\title{
Existence of Meromorphic Solutions of First-Order Difference Equations
}

\author{
Risto Korhonen ${ }^{1} \cdot$ Yueyang Zhang ${ }^{2,3}$
}

Received: 29 May 2018 / Revised: 2 April 2019 / Accepted: 9 October 2019 /

Published online: 17 December 2019

(c) The Author(s) 2019

\section{Abstract}

It is shown that if

$$
f(z+1)^{n}=R(z, f),
$$

where $R(z, f)$ is rational in $f$ with meromorphic coefficients and $\operatorname{deg}_{f}(R(z, f))=n$, has an admissible meromorphic solution, then either $f$ satisfies a difference linear or Riccati equation with meromorphic coefficients, or the equation above can be transformed into one in a list of ten equations with certain meromorphic or algebroid coefficients. In particular, if $f(z+1)^{n}=R(z, f)$, where the assumption $\operatorname{deg}_{f}(R(z, f))=n$ has been discarded, has rational coefficients and a transcendental meromorphic solution $f$ of hyper-order $<1$, then either $f$ satisfies a difference linear or Riccati equation with rational coefficients, or the equation above can be transformed into one in a list of five equations which consists of four difference Fermat equations and one equation which is a special case of the symmetric QRT map. Solutions to all of these equations are presented in terms of Weierstrass or Jacobi elliptic functions, or in terms of meromorphic functions that are solutions to a difference Riccati equation. This provides a natural difference analogue of Steinmetz' generalization of Malmquist's theorem.

Keywords Difference equation - Meromorphic solution - Malmquist's theorem · Nevanlinna theory

Mathematics Subject Classification Primary 39A10; Secondary 30D35 · 39A12

Communicated by Stephan Ruscheweyh.

The first author would like to thank the partial support of the Academy of Finland Grant 286877. The second author was supported in part by NSFC (No. 11371225), the China Scholarship Council and the Fundamental Research Funds for the Central Universities (FRF-TP-19-055A1).

Extended author information available on the last page of the article 


\section{Introduction}

Global existence of large classes of meromorphic solutions is a rare property for a differential equation to have. According to a classical result due to Malmquist [14], if the first-order differential equation

$$
f^{\prime}=R(z, f)
$$

where $R(z, f)$ is rational in both arguments, has a transcendental meromorphic solution, then (1.1) reduces to the Riccati equation

$$
f^{\prime}=a_{2} f^{2}+a_{1} f+a_{0}
$$

with rational coefficients $a_{0}, a_{1}$ and $a_{2}$. Generalizations of Malmquist's theorem for the equation

$$
\left(f^{\prime}\right)^{n}=R(z, f), \quad n \in \mathbb{N},
$$

have been given by Yosida [31] and Laine [12]. Steinmetz [24] and Bank and Kaufman [3] proved that if (1.3) has rational coefficients and a transcendental meromorphic solution, then by a suitable Möbius transformation, (1.3) can be either mapped to (1.2) or to one of the equations in the following list:

$$
\begin{aligned}
& \left(f^{\prime}\right)^{2}=a(f-b)^{2}\left(f-\tau_{1}\right)\left(f-\tau_{2}\right), \\
& \left(f^{\prime}\right)^{2}=a\left(f-\tau_{1}\right)\left(f-\tau_{2}\right)\left(f-\tau_{3}\right)\left(f-\tau_{4}\right), \\
& \left(f^{\prime}\right)^{3}=a\left(f-\tau_{1}\right)^{2}\left(f-\tau_{2}\right)^{2}\left(f-\tau_{3}\right)^{2}, \\
& \left(f^{\prime}\right)^{4}=a\left(f-\tau_{1}\right)^{2}\left(f-\tau_{2}\right)^{3}\left(f-\tau_{3}\right)^{3}, \\
& \left(f^{\prime}\right)^{6}=a\left(f-\tau_{1}\right)^{3}\left(f-\tau_{2}\right)^{4}\left(f-\tau_{3}\right)^{5},
\end{aligned}
$$

where $a$ and $b$ are rational functions and $\tau_{1}, \ldots, \tau_{4}$ are distinct constants.

The existence of globally meromorphic solutions is somewhat more common in the case of difference equations as compared to differential equations. It was shown by Shimomura [23] that the difference equation

$$
f(z+1)=P(f(z))
$$

where $P(f(z))$ is a polynomial in $f(z)$ with constant coefficients, always has a nontrivial entire solution. On the other hand, Yanagihara [28] showed that the difference equation

$$
f(z+1)=R(f(z)),
$$

where $R(f(z))$ is rational in $f(z)$ having constant coefficients, has a nontrivial meromorphic solution no matter how $R$ is chosen. Yanagihara [29] also considered higher order equations and showed, for instance, that the difference equation

$\alpha_{n} f(z+n)+\alpha_{n-1} f(z+n-1)+\cdots+\alpha_{1} f(z+1)=R(f(z)), \quad \alpha_{1}, \ldots, \alpha_{n} \in \mathbb{C}$, 
has a nontrivial meromorphic solution if the degree $p$ of the numerator $P(f(z))$ of the rational function $R(f(z))$ satisfies $p \geq q+2$, where $q$ is the degree of the denominator $Q(f(z))$, and $P(f(z))$ and $Q(f(z))$ have no common factors.

Ablowitz, Halburd and Herbst [1] suggested that the existence of sufficiently many finite-order meromorphic solutions of a difference equation is a good difference analogue of the Painlevé property for differential equations. An ordinary differential equation is said to have the Painlevé property when all solutions are single-valued around all movable singularities. They showed, for instance, that if the difference equation

$$
f(z+1)+f(z-1)=R(z, f(z)),
$$

where $R(z, f(z))$ is rational in both arguments, has a transcendental meromorphic solution of finite order, then $\operatorname{deg}_{f}(R(z, f(z))) \leq 2$. Their results are consistent with Yanagihara's work on the first-order equation [28] in which he proved that if

$$
f(z+1)=R(z, f(z))
$$

where $R(z, f(z))$ is rational in both arguments, has a transcendental meromorphic solution of hyper-order strictly less than one, then $\operatorname{deg}_{f}(R(z, f(z)))=1$ and thus (1.5) reduces into the difference Riccati equation. This is a natural difference analogue of Malmquist's 1913 result on differential equations. Halburd and the first author [7] showed that if (1.4), where the right-hand side has meromorphic coefficients, has an admissible meromorphic solution $f$ of finite order, then either $f$ satisfies a difference Riccati equation, or a linear transformation of (1.4) reduces it to one in a short list of difference equations that consists solely of difference Painlevé equations and equations related to them, linear equations, and linearizable equations. The finite-order condition was relaxed into hyper-order strictly less than one by Halburd, the first author, and Tohge [9].

The purpose of this paper is to present a natural difference analogue of Steinmetz's generalization of Malmquist's theorem. We will show that if the difference equation

$$
f(z+1)^{n}=R(z, f)
$$

with rational coefficients has a transcendental meromorphic solution $f$ of hyper-order $<1$, then either $f$ satisfies a difference linear or Riccati equation

$$
\begin{aligned}
f(z+1) & =a_{1}(z) f(z)+a_{2}(z), \\
f(z+1) & =\frac{b_{1}(z) f(z)+b_{2}(z)(z)}{f(z)+b_{3}(z)},
\end{aligned}
$$

where $a_{i}(z)$ and $b_{j}(z)$ are rational functions, or, by implementing a transformation $f \rightarrow \alpha f$ or $f \rightarrow 1 /(\alpha f)$ with an algebraic function $\alpha$ of degree at most 2, (1.6) reduces into one of the following equations:

$$
f(z+1)^{2}=1-f(z)^{2}
$$




$$
\begin{aligned}
& f(z+1)^{2}=1-\left(\frac{\delta(z) f(z)-1}{f(z)-\delta(z)}\right)^{2}, \\
& f(z+1)^{2}=1-\left(\frac{f(z)+3}{f(z)-1}\right)^{2}, \\
& f(z+1)^{2}=\frac{f(z)^{2}-\kappa^{2}}{f(z)^{2}-1}, \\
& f(z+1)^{3}=1-f(z)^{-3},
\end{aligned}
$$

where $\delta(z)(\not \equiv \pm 1)$ is an algebraic function of degree 2 at most and $\kappa^{2} \neq 0,1$ is a constant. Equations (1.7) and (1.8) are the linear and the difference Riccati equation, respectively, Eqs. (1.9)-(1.11) and (1.13) are difference Fermat equations, while (1.12) is a special case of the symmetric Quispel-Roberts-Thompson (QRT) map. We will present finite-order meromorphic solutions to autonomous versions of all of these equations in terms of Weierstrass or Jacobi elliptic functions, or in terms of meromorphic functions that are solutions to a difference Riccati equation, in Sect. 3 below.

Under the condition that the meromorphic solution $f$ of (1.6) is of hyper-order less than 1 , it can actually be shown that $\operatorname{deg}_{f}(R(z, f))=n$ by using an asymptotic relation between the Nevanlinna characteristics (see Sect. 2 below) $T(r, f(z+1))$ and $T(r, f(z))$ from [9], and the Valiron-Mohon'ko identity [15,26] (see also [13]). By discarding the assumption that the meromorphic solution is of hyper-order $<1$, and considering the more general case of admissible meromorphic solutions of (1.6) with meromorphic coefficients and such that $\operatorname{deg}_{f}(R(z, f))=n$, it follows either that $f$ satisfies (1.7) or (1.8), or (1.6) can be transformed into one of the Eqs. (1.9)(1.13), but now with meromorphic coefficients $a_{i}(z)$ and $b_{j}(z)$, an algebroid function $\delta(z)=\delta_{2}(z)$ of degree at most 2 and with $\kappa^{2}=\kappa_{1}(z)^{2}$ being a meromorphic periodic function of period 1, or (1.6) becomes one of the following equations:

$$
\begin{aligned}
& f(z+1)^{2}=\delta_{1}(z)\left(f(z)^{2}-1\right), \\
& f(z+1)^{2}=\delta_{3}(z)\left(1-f(z)^{-2}\right), \\
& f(z+1)^{2}=\frac{\kappa_{2}(z+1)^{2} f(z)^{2}-1}{f(z)^{2}-1}, \\
& f(z+1)^{2}=\theta \frac{f(z)^{2}-\kappa_{3}(z) f(z)+1}{f(z)^{2}+\kappa_{3}(z) f(z)+1}, \\
& f(z+1)^{3}=1-f(z)^{3},
\end{aligned}
$$

where $\theta= \pm 1$ and $\delta_{1}(z), \delta_{3}(z), \kappa_{2}(z)^{2}, \kappa_{3}(z)^{2}$ are meromorphic functions each of which satisfies a certain difference equation, see Sect. 3 below. In particular, if the coefficients of (1.6) are rational functions, then $\theta= \pm 1$ and $\delta_{1}(z), \delta_{3}(z), \kappa_{2}(z)^{2}$, $\kappa_{3}(z)^{2}$ are all constants. We will show that meromorphic solutions of autonomous versions of (1.14)-(1.18) can be characterized by Weierstrass or Jacobi elliptic functions composed with certain entire functions, but none of them is of hyper-order $<1$. 
The remainder of this paper is organized in the following way. Section 2 contains the necessary notation and preliminary results needed to prove our main theorem given in Sect. 3. Section 3 also contains a discussion of the Eqs. (1.9)-(1.18). In particular, we will show that either their autonomous versions have meromorphic solutions expressible in terms of Weierstrass or Jacobi elliptic functions, or solutions of these equations can be explicitly expressed in terms of meromorphic functions that are solutions of certain difference Riccati equations. The proof of our main result, Theorem 2 below, has been split into the three remaining Sects. 4-6.

\section{Preliminaries}

In this section, we introduce some preliminaries for the proof of our main results. In the following, a meromorphic function is always meromorphic on the whole complex plane $\mathbb{C}$. Let $\mathcal{M}$ denote the field of meromorphic functions, and let $f(z) \in \mathcal{M}$. We assume that the readers are familiar with the basic notation and fundamental results of Nevanlinna theory [10], such as $m(r, f), N(r, f), T(r, f)$, and Nevanlinna's main theorems. Moreover, we use the notation $\sigma(f)$ to denote the order of growth of $f(z)$, which is defined to be

$$
\sigma(f)=\limsup _{r \rightarrow \infty} \frac{\log T(r, f)}{\log r},
$$

and, if $f(z)$ is of order $\sigma(f)=\infty$, we use the notation $\sigma_{2}(f)$ to denote the hyper-order of $f(z)$, which is defined to be

$$
\sigma_{2}(f)=\limsup _{r \rightarrow \infty} \frac{\log \log T(r, f)}{\log r} .
$$

Let $S(r, f)$ denote any quantity that satisfies the condition $S(r, f)=o(1) T(r, f)$, $r \rightarrow \infty$, outside of a possible exceptional set of finite logarithmic measure. For a meromorphic function $c(z) \in \mathcal{M}$, if $c(z)$ satisfies $T(r, c(z))=S(r, f)$, then $c(z)$ is said to be small compared to $f(z)$. For example, all rational functions are small with respect to any transcendental meromorphic function. Denote by $\mathcal{S}(f)$ the field of all small functions of $f(z)$, i.e.,

$$
\mathcal{S}(f)=\{c(z) \in \mathcal{M}: T(r, c(z))=S(r, f)\} .
$$

Set $\hat{\mathcal{S}}(f)=\mathcal{S}(f) \cup\{\infty\}$. A meromorphic solution $f(z)$ of a differential equation is called admissible if all coefficients of the equation are in $\mathcal{S}(f)$ [13]. This definition is very natural for differential equations, but for difference equations to be studied in this paper we need to be slightly more careful due to the possible effect of the shift operation on the growth of the solution and of the coefficients. We define the following set for small functions of a meromorphic function $f(z)$ :

$$
\mathcal{S}^{\prime}(f)=\{c(z) \in \mathcal{M}: T(r, c(z+n))=S(r, f(z+n)), n \in \mathbb{N}\},
$$


and in what follows we say that a meromorphic solution $f(z)$ of a difference equation is admissible if all coefficients of the equation are in $\mathcal{S}^{\prime}(f)$. If the hyper-order of $f(z)$ is less than one, then by [9, Lemma 8.3] it follows that

$$
T(r, f(z+1))=T(r, f)+S(r, f),
$$

and so in this case shifting a difference equation does not affect the admissibility of solutions.

The fundamental results of Nevanlinna's theory are the First Main Theorem and the Second Main Theorem. Nevanlinna's Second Main Theorem can be simplified into the following form:

$$
(q-2) T(r, f) \leq \sum_{i=1}^{q} \bar{N}\left(r, a_{i}, f\right)+S(r, f),
$$

where $q \geq 3, a_{i} \in \mathbb{C} \cup\{\infty\}$, and $\bar{N}\left(r, a_{i}, f\right)$ is the truncated counting function for $a_{i}$-points (poles) of $f(z)$, and the error term $S(r, f)=O(\log r), r \rightarrow \infty$, when $f(z)$ is of finite order and $S(r, f)=O(\log r T(r, f)), r \rightarrow \infty$, outside a possible set of finite linear measure when $f(z)$ is of infinite order. Yamanoi [27, Corollary 1] generalized Nevanlinna's Second Main Theorem by proving that, for any collection of functions $c_{i}(z) \in \hat{\mathcal{S}}(f), i=1, \ldots, q$, of $f(z)$, the inequality

$$
(q-2-\varepsilon) T(r, f) \leq \sum_{i=1}^{q} \bar{N}\left(r, c_{i}, f\right), \quad \text { for all } \varepsilon>0
$$

holds outside an exceptional set $E=E(\varepsilon) \subset(0, \infty)$ satisfying $\int_{E} d \log \log r<\infty$. The inequality (2.3) implies a generalization of the defect relation for small functions of $f(z)$ [27, Corollary 1] and that

$$
\sum_{i=1}^{q} \Theta\left(c_{i}, f\right) \leq 2
$$

where the quantity $\Theta(c, f), c=c(z) \in \hat{\mathcal{S}}(f)$, is defined as

$$
\Theta(c, f)=1-\limsup _{r \rightarrow \infty} \frac{\bar{N}(r, c, f)}{T(r, f)} .
$$

Recall that a value $a$ is said to be a completely ramified value of $f(z)$ when $f(z)-a=0$ has no simple roots. In what follows, we say that $c(z) \in \hat{\mathcal{S}}(f)$ is a completely ramified small function of $f(z)$ when $f(z)-c(z)=0$ has at most $S(r, f)$ many simple roots and that $c(z) \in \hat{\mathcal{S}}(f)$ is a Picard exceptional small function of $f(z)$ when $N(r, c, f)=S(r, f)$. A nonconstant meromorphic function $f(z)$ can have at most two Picard exceptional small functions. Moreover, by (2.4) and the First Main Theorem, we have the following result. 
Theorem 1 A nonconstant meromorphic function $f(z)$ can have at most four completely ramified small functions.

In what follows, when considering a meromorphic solution $f$ of (1.6), we will do a transformation to $f$ using some algebroid functions and end up in a situation such that the considered function has some finite-sheeted branching. The classical version of Nevanlinna theory introduced above cannot be used to handle this situation. In this case, we need the algebroid version of Nevanlinna theory introduced by Selberg [2022], Ullrich [25] and Valiron [26] (see also [11]), which studies meromorphic functions on a finitely sheeted Riemann surface. All algebroid functions we need to consider in this paper are either small functions with respect to an admissible meromorphic solution $f$ of (1.6), or can be obtained from it by a Möbius transformation with small algebroid coefficients. Such functions could be described as "almost meromorphic" in the sense of Nevanlinna theory, since the presence of branch points actually only affects the small error term $S(r, f)$ in any of the estimates involving Nevanlinna functions. Correspondingly, $T(r, f)$ and $N(r, f)$ will denote the characteristic and counting functions of a finite-sheeted algebroid function $f$, and similarly with the rest of the Nevanlinna functions involving $f$.

For simplicity, from now on we will use the suppressed notation: $f=f(z), \bar{f}=$ $f(z+1)$, and $\underline{f}=f(z-1)$ for a meromorphic, or algebroid, function $f(z)$.

\section{Extension of the Difference Malmquist Theorem}

As mentioned in the introduction, from Eq. (1.5) Yanagihara [28] obtained a difference analogue of Malmquist's theorem. Let $n \in \mathbb{N}$. Nakamura and Yanagihara [16], and Yanagihara [30], considered meromorphic solutions of a more general case,

$$
\bar{f}^{n}=P(z, f),
$$

where $P(z, f)$ is a polynomial in $f$ with constants as coefficients, and, in particular, Yanagihara [30] showed that if (3.1) has a meromorphic solution of finite order, then either $\bar{f}=A f+B$ for some constants $A, B$ or (3.1) reduces to $\bar{f}^{2}=1-f^{2}$. We consider the difference equation

$$
\bar{f}^{n}=R(z, f),
$$

where $R(z, f)$ is rational in $f$ having meromorphic coefficients. If Eq. (3.2) has an admissible meromorphic solution $f$ such that the hyper-order $\sigma_{2}(f)$ of $f$ satisfies $\sigma_{2}(f)<1$, then, by (3.2), it follows from (2.1) and the Valiron-Mohon'ko identity $[15,26]$ (see also [13]) that

$$
\begin{aligned}
\operatorname{deg}_{f}(R(z, f)) T(r, f) & =T(r, R(z, f))+S(r, f) \\
& =T\left(r, \bar{f}^{n}\right)+S(r, f) \\
& =n T(r, \bar{f})+S(r, f) \\
& =n T(r, f)+S(r, f),
\end{aligned}
$$


which implies that $\operatorname{deg}_{f}(R(z, f))=n$. Assume that $\operatorname{deg}_{f}(R(z, f))=n$ and that all the coefficients of (3.2) are in $\mathcal{S}^{\prime}(f)$. Then the characteristic function of $f(z)$ satisfies $T(r, f(z+j))=T(r, f(z))+S(r, f), j \in \mathbb{N}$. Under these conditions, but discarding the assumption $\sigma_{2}(f)<1$, we now use Nevanlinna theory to simplify Eq. (3.2). Our main result, which is an extension of the result on (3.1) of Yanagihara [30], is stated as follows.

Theorem 2 Let $n \in \mathbb{N}$. If the difference equation (3.2) with $\operatorname{deg}_{f}(R(z, f))=n$ has an admissible meromorphic solution, then either $f$ satisfies a difference linear or Riccati equation:

$$
\begin{aligned}
& \bar{f}=a_{1} f+a_{2}, \\
& \bar{f}=\frac{b_{1} f+b_{2}}{f+b_{3}},
\end{aligned}
$$

where $a_{i}, b_{j}$ are small meromorphic functions; or, by a transformation $f \rightarrow \alpha f$ or $f \rightarrow 1 /(\alpha f)$ with a small algebroid function $\alpha$ of degree at most 3, (3.2) reduces to one of the following equations:

$$
\begin{aligned}
& \bar{f}^{2}=1-f^{2}, \\
& \bar{f}^{2}=\delta_{1}\left(f^{2}-1\right), \\
& \bar{f}^{2}=1-\left(\frac{\delta_{2} f-1}{f-\delta_{2}}\right)^{2}, \\
& \bar{f}^{2}=\delta_{3}\left(1-f^{-2}\right), \\
& \bar{f}^{2}=1-\left(\frac{f+3}{f-1}\right)^{2}, \\
& \bar{f}^{2}=\frac{f^{2}-\kappa_{1}^{2}}{f^{2}-1} \\
& \bar{f}^{2}=\frac{\kappa_{2}^{2} f^{2}-1}{f^{2}-1}, \\
& \bar{f}^{2}=\theta \frac{f^{2}-\kappa_{3} f+1}{f^{2}+\kappa_{3} f+1}, \\
& \bar{f}^{3}=1-f^{3}, \\
& \bar{f}^{3}=1-f^{-3},
\end{aligned}
$$

where $\theta= \pm 1, \delta_{2} \not \equiv \pm 1$ is a small algebroid function of degree at most 2 and $\delta_{1}$, $\delta_{3}, \kappa_{1}^{2}, \kappa_{2}^{2}, \kappa_{3}^{2}$ are all small meromorphic functions satisfying $\bar{\delta}_{1}\left(\delta_{1}+1\right)+1=0$, $\bar{\delta}_{3} \delta_{3}=\bar{\delta}_{3}+\delta_{3}, \bar{\kappa}_{1}^{2}=\kappa_{1}^{2}, \bar{\kappa}_{2}^{2} \kappa_{2}^{2}=1$, and $\bar{\kappa}_{3}^{2}\left(\kappa_{3}^{2}-4\right)=2(1-\theta) \kappa_{3}^{2}-8(1+\theta)$, respectively.

Assume that in Theorem 2 all the coefficients of (3.2) are rational functions. Then it is seen that the coefficients $\delta_{1}, \delta_{3}, \kappa_{1}^{2}, \kappa_{2}^{2}, \kappa_{3}^{2}$ are all constants. In this case, it is easy 
to show by iteration and substitution that solutions to (3.5), (3.6), (3.8), (3.10), (3.11), (3.13), and (3.14) are all periodic functions, which implies that for these equations the coefficient $\alpha$ in the transformation $f \rightarrow \alpha f$ or $f \rightarrow 1 /(\alpha f)$ has no branch points. Also, from the proof of Theorem 2 we see that when obtaining (3.9) and (3.12), the coefficient $\alpha$ in the transformation $f \rightarrow \alpha f$ or $f \rightarrow 1 /(\alpha f)$ is rational. Thus the algebraic case of $\alpha$ can only occur when obtaining Eq. (3.7).

In the rational coefficients case of (3.2), solutions to (3.13) and (3.14) can be characterized by Weierstrass elliptic functions, solutions to Eqs. (3.6), (3.8), (3.10)(3.12) can be characterized by Jacobi elliptic functions, and Eqs. (3.5), (3.7) and (3.9) can be explicitly solved in terms of functions that are solutions of certain difference Riccati equations, as is shown below.

Equations (3.5), (3.7), (3.9), (3.13), and (3.14) are so-called Fermat difference equations. In general, a Fermat equation is a function analogue of the Fermat diophantine equation $x^{n}+y^{n}=1$; i.e., $h(z)^{n}+g(z)^{n}=1$, where $n \geq 2$ is an integer. Meromorphic solutions to Fermat equations have been clearly characterized, see $[2,5,6]$, for example. In particular, when $n=3$, all meromorphic solutions can be represented as $h=H(\varphi), g=\eta G(\varphi)=\eta H(-\varphi)=H\left(-\eta^{2} \varphi\right)$, where $\varphi=\varphi(z)$ is an entire function and $\eta$ is a cubic root of 1 , and

$$
H(z)=\frac{1+\wp^{\prime}(z) / \sqrt{3}}{2 \wp(z)}, \quad G(z)=\frac{1-\wp^{\prime}(z) / \sqrt{3}}{2 \wp(z)},
$$

is a pair of solutions of the Fermat equation with $\wp(z)$ being the particular Weierstrass elliptic function satisfying $\wp^{\prime}(z)^{2}=4 \wp(z)^{3}-1$. Now, for Eq. (3.13) the solution takes the form $f=H(\varphi), \bar{f}=\eta G(\varphi)=H\left(-\eta^{2} \varphi\right)$. It follows that $\bar{\varphi}=-\eta^{2} \varphi+\omega$, where $\omega$ is a period of $\wp(\varphi)$. This implies that $\varphi=v\left(-\eta^{2}\right)^{z}+\omega /\left(1+\eta^{2}\right)$, where $v$ is an arbitrary periodic entire function of period 1 , and thus $\varphi$ is a transcendental entire function of order at least 1 , of mean type. From (3.13) we have $T(r, \bar{f})=T(r, f)+O(1)$. Moreover, we have $\eta f+\bar{f}=\eta / \wp(\varphi)$, which yields $T(r, \wp(\varphi)) \leq 2 T(r, f)+O(1)$. By taking the derivatives on both sides of $\eta f+\bar{f}=\eta / \wp(\varphi)$ and combining it with the resulting equation, we get

$$
\varphi^{\prime 2}=\left(\frac{(\eta f+\bar{f})^{\prime}}{\eta f+\bar{f}}\right)^{2} \cdot \frac{\wp(\varphi)^{2}}{4 \wp(\varphi)^{3}-1}
$$

Since $\wp(\varphi)$ satisfies $\wp^{\prime}(\varphi)^{2}=4 \wp(\varphi)^{3}-1$, we see that $\wp(\varphi)$, as a function of $z$, is twofold ramified over poles and each of $\vartheta_{i}, i=1,2,3$, where $\vartheta_{i}$ are the three cubic roots of 1/4. Then the First Main Theorem and the Second Main Theorem (2.2) yield that $m(r, \wp(\varphi))+\sum_{i=1}^{3} m\left(r, \frac{1}{\wp(\varphi)-\vartheta_{i}}\right) \leq O(\log r T(r, \wp(\varphi)))$. Thus, by taking the proximity functions on both sides of $(3.16)$, we get

$$
\begin{aligned}
m\left(r, \varphi^{\prime 2}\right) \leq & O(\log r T(r, f))+2 m(r, \wp(\varphi)) \\
& +\sum_{i=1}^{3} m\left(r, \frac{1}{\wp(\varphi)-\vartheta_{i}}\right)+O(1)=O(\log r T(r, f))+O(1) .
\end{aligned}
$$


Then, $T\left(r, \varphi^{\prime 2}\right)=m\left(r, \varphi^{\prime 2}\right) \leq O(\log r T(r, f))+O(1)$, which together with [13, Lemma 1.1.1] yields that $\sigma_{2}(f) \geq 1$, and so Eq. (3.13) cannot have meromorphic solutions of hyper-order less than 1 .

We now show that the autonomous versions of (3.6), (3.8), (3.11), and (3.12) cannot admit meromorphic solutions of hyper-order $<1$ either. We only need to consider Eqs. (3.6) and (3.11) since (3.8) and (3.12) can be transformed into (3.6) or (3.11) in the following way. For Eq. (3.8), $\delta_{3}=2$, and we see that $f^{2}-1$ is written as $f^{2}-1=-(i \bar{f} f / \sqrt{2})^{2}=-f_{0}^{2}$. Then we have $T(r, f)=T\left(r, f_{0}\right)+O(1)$, and by substituting $f^{2}=-f_{0}^{2}+1$ into (3.8) we get the autonomous case of Eq. (3.11). For Eq. (3.12), if we let $w=f+1 / f$, then $w$ satisfies $T(r, w)=2 T(r, f)+O(1)$ by the Valiron-Mohon'ko identity [15,26] (see also [13]), and it follows that

$$
\bar{f}^{2}=\theta \frac{w-\kappa_{3}}{w+\kappa_{3}}, \quad \frac{1}{\bar{f}^{2}}=\theta \frac{w+\kappa_{3}}{w-\kappa_{3}} .
$$

The above two equations yield

$$
\bar{w}^{2}=\frac{2(\theta+1) w^{2}+2(\theta-1) \kappa_{3}^{2}}{w^{2}-\kappa_{3}^{2}} .
$$

If $\theta=1$, then we have $\bar{\kappa}_{3}^{2}\left(\kappa_{3}^{2}-4\right)=-16$, and by doing a transformation $w \rightarrow$ $\kappa_{3} / w$ we get $\bar{w}^{2}=d_{1}\left(w^{2}-1\right)$, which is the Eq. (3.6) since $d_{1}=-\bar{\kappa}_{3}^{2} / 4$ satisfies $\bar{d}_{1}\left(d_{1}+1\right)+1=0$; if $\theta=-1$, then we have $\bar{\kappa}_{3}^{2}\left(\kappa_{3}^{2}-4\right)=4 \kappa_{3}^{2}$, and by doing a transformation $w \rightarrow \kappa_{3} / w$ we get $\bar{w}^{2}=d_{2}\left(1-w^{-2}\right)$, which is the Eq. (3.8) since $d_{2}=\bar{\kappa}_{3}^{2} / 4$ satisfies $\bar{d}_{2} d_{2}=\bar{d}_{2}+d_{2}$.

Recall that the Jacobi elliptic function $\operatorname{sn}(z, k)$ with the elliptic modulus $k \in(0,1)$ satisfies the differential equation $\operatorname{sn}^{\prime}(z)^{2}=\left(1-\operatorname{sn}(z)^{2}\right)\left(1-k^{2} \operatorname{sn}(z)^{2}\right)$. For Eq. (3.6), $\delta_{1}$ is now a cubic root of 1 , and we see that $f$ is twofold ramified over \pm 1 . We write $\delta_{1}=\eta^{2}$, where $\eta$ is the cubic root of 1 satisfying $\eta^{2}\left(\eta^{2}+1\right)+1=\eta^{2}+\eta+1=0$. Let $z_{0}$ be such that $f\left(z_{0}\right)= \pm i \eta$, by (3.6) we have $f\left(z_{0}+1\right)= \pm 1$, and so $f$ is also twofold ramified at $\pm i \eta$-points. Let $\varrho_{1}=\exp (7 i \pi / 12)$, and set $\tau_{1}=\left(1-\varrho_{1}\right) /\left(1+\varrho_{1}\right)$. Then

$$
g_{1}=\tau_{1} \frac{f+\varrho_{1}}{f-\varrho_{1}}
$$

is twofold ramified over each of $\pm 1, \pm \tau_{1}^{2}$, where $-\tau_{1}^{2}=\tan (7 \pi / 24)^{2}>1$. Let $\operatorname{sn}(\phi)=\operatorname{sn}\left(\phi,-1 / \tau_{1}^{2}\right)$ and $\phi_{0}$ be such that $\operatorname{sn}\left(\phi_{0}\right) \neq \pm 1, \pm \tau_{1}^{2}$. Letting $z_{0}$ be such that $g_{1}\left(z_{0}\right)=\operatorname{sn}\left(\phi_{0}\right)$, it follows that there is a neighborhood $U$ of $z_{0}$ such that $\phi_{1}(z)=$ $\mathrm{sn}^{-1}\left(g_{1}(z)\right)$ is defined and holomorphic in $U$. By following the reasoning in the proof of [30, Lemma 4.1], we know that $\phi_{1}(z)$ can be continued analytically throughout the complex plane to an entire function. Therefore, $g_{1}$ is written as $g_{1}(z)=\operatorname{sn}\left(\phi_{1}(z)\right)$, where $\operatorname{sn}\left(\phi_{1}\right)=\operatorname{sn}\left(\phi_{1},-1 / \tau_{1}^{2}\right)$ satisfies the differential equation $\operatorname{sn}^{\prime}\left(\phi_{1}\right)^{2}=(1-$ $\left.\operatorname{sn}\left(\phi_{1}\right)^{2}\right)\left(1-\operatorname{sn}\left(\phi_{1}\right)^{2} / \tau_{1}^{4}\right)$. By taking the derivative of $g_{1}$ and combining it with the 
resulting equation, we get

$$
\phi_{1}^{\prime 2}=\frac{\operatorname{sn}\left(\phi_{1}\right)^{2}}{\operatorname{sn}^{\prime}\left(\phi_{1}\right)^{2}} \cdot\left(\frac{g_{1}^{\prime}}{g_{1}}\right)^{2}=\frac{g_{1}^{2}}{\left(1-g_{1}^{2}\right)\left(1-g_{1}^{2} / \tau_{1}^{4}\right)} \cdot\left(\frac{g_{1}^{\prime}}{g_{1}}\right)^{2} .
$$

Since $g_{1}$ is twofold ramified over each of $\pm 1, \pm \tau_{1}^{2}$, the First Main Theorem and the Second Main Theorem (2.2) yield that $2 m\left(r, g_{1}\right)+\sum_{i=1}^{4} m\left(r, \frac{1}{g_{1}-l_{i}}\right) \leq$ $O\left(\log r T\left(r, g_{1}\right)\right), \iota_{i}= \pm 1, \pm \tau_{1}^{2}$. Note that $T(r, f)=T\left(r, g_{1}\right)+O(1)$. Now taking the proximity function on both sides of (3.17) gives $T\left(r, \phi_{1}^{\prime 2}\right)=m\left(r, \phi_{1}^{\prime 2}\right) \leq$ $O(\log r T(r, f))+O(1)$, which together with [13, Lemma 1.1.1] yields $\sigma\left(\phi_{1}\right) \leq$ $\sigma_{2}(f)$. Suppose that $\sigma_{2}(f)<1$. By iterating (3.6) we obtain $f(z+3)^{2}=f(z)^{2}$, and so $f(z+6)=f(z)$ and it follows that $\operatorname{sn}\left(\phi_{1}(z+6)\right)=\operatorname{sn}\left(\phi_{1}(z)\right)$ giving $\phi_{1}(z+6)=\phi_{1}(z)+K_{1}$ with a period $K_{1}$ of $\operatorname{sn}\left(\phi_{1}\right)$, which is possible only when $\phi_{1}$ is a polynomial of degree 1 since $\phi_{1}^{\prime}(z+6)=\phi_{1}^{\prime}(z)$. This implies that $f$ is of finite order. But according to [30, Lemma 9.1], (3.6) cannot admit any meromorphic solutions of finite order when $\delta_{1}$ is a constant. Therefore, (3.6) cannot admit any meromorphic solutions of hyper-order strictly less than 1 when $\delta_{1}$ is a constant.

For Eq. (3.11), if $\kappa_{2}$ is a constant, then $\kappa_{2}^{2}=-1$ and we see that $f$ is twofold ramified over each of $\pm i, \pm 1$. Let $\varrho_{2}=\exp (i \pi / 4)$, and set $\tau_{2}=\left(1+\varrho_{2}\right) /\left(1-\varrho_{2}\right)$. Then

$$
g_{2}=\tau_{2} \frac{f-\varrho_{2}}{f+\varrho_{2}}
$$

is twofold ramified over each of $\pm \tau_{2}^{2}, \pm 1$, where $-\tau_{2}^{2}=(\sqrt{2}+1)^{2}>1$. By similar arguments as above, there exists an entire function $\phi_{2}(z)$ such that $g_{2}$ is written as $g_{2}(z)=\operatorname{sn}\left(\phi_{2}(z)\right)$, where $\operatorname{sn}\left(\phi_{2}\right)=\operatorname{sn}\left(\phi_{2},-1 / \tau_{2}^{2}\right)$. Thus

$$
f=-\varrho_{2} \frac{\operatorname{sn}\left(\phi_{2}\right)+\tau_{2}}{\operatorname{sn}\left(\phi_{2}\right)-\tau_{2}} .
$$

Suppose that $\sigma_{2}(f)<1$. Then we have $\sigma\left(\phi_{2}^{\prime}\right) \leq \sigma_{2}(f)<1$. Iterating (3.11) gives $f(z+4)^{2}=f(z)^{2}$. Therefore, we have $f(z+8)=f(z)$ and then $\operatorname{sn}\left(\phi_{2}(z+8)\right)=$ $\operatorname{sn}\left(\phi_{2}(z)\right)$, giving $\phi_{2}(z+8)=\phi_{2}(z)+K_{2}$ with a period $K_{2}$ of $\operatorname{sn}\left(\phi_{2}\right)$, which is possible only when $\phi_{2}$ is a polynomial of degree 1 since $\phi_{2}^{\prime}(z+8)=\phi_{2}^{\prime}(z)$. Write $\phi_{2}(z)=C z+D$ for two constants $C, D$. Supposing that $\operatorname{sn}\left(\phi_{2}\left(z_{0}\right)\right)=1$; we then have $f\left(z_{0}\right)=1$. By (3.11) we have $f\left(z_{0}+1\right)=\infty$ and it follows that $\operatorname{sn}\left(\phi_{2}\left(z_{0}+1\right)\right)=\tau_{2}$. On the other hand, if $\operatorname{sn}\left(\phi_{2}\left(z_{1}\right)\right)=\tau_{2}^{2}$, then $f\left(z_{1}\right)=-1$ and it follows from (3.11) that $f\left(z_{1}+1\right)=\infty$ and hence $\operatorname{sn}\left(\phi_{2}\left(z_{1}+1\right)\right)=\tau_{2}$. Thus, $\operatorname{sn}\left(\phi_{2}\left(z_{0}+1\right)\right)=\operatorname{sn}\left(\phi_{2}\left(z_{1}+1\right)\right)$, which implies that $\phi_{2}\left(z_{0}\right)-\phi_{2}\left(z_{1}\right)=C\left(z_{0}-z_{1}\right)=\phi_{2}\left(z_{0}+1\right)-\phi_{2}\left(z_{1}+1\right)=K_{2}$ with a period $K_{2}$, but $\operatorname{sn}\left(\phi_{2}\left(z_{0}\right)\right) \neq \operatorname{sn}\left(\phi_{2}\left(z_{1}\right)\right)$, a contradiction. Therefore, (3.11) cannot admit any meromorphic solutions of hyper-order strictly less than 1 when $\kappa_{2}$ is a constant.

From the above discussions, we obtain the following corollary, which is a natural difference analogue of Steinmetz's generalization [24] of Malmquist's 1913 result on differential equations. 
Corollary 1 Let $n \in \mathbb{N}$. If the difference equation (3.2) with rational coefficients has a transcendental meromorphic solution of hyper-order strictly less than 1, then either $f$ satisfies (3.3) or (3.4) with rational coefficients or, by a transformation $f \rightarrow \alpha f$ or $f \rightarrow 1 /(\alpha f)$ with an algebraic function $\alpha$ of degree at most 2, (3.2) reduces to one of the following equations:

$$
\begin{aligned}
& \bar{f}^{2}=1-f^{2}, \\
& \bar{f}^{2}=1-\left(\frac{\delta f-1}{f-\delta}\right)^{2}, \\
& \bar{f}^{2}=1-\left(\frac{f+3}{f-1}\right)^{2}, \\
& \bar{f}^{2}=\frac{f^{2}-\kappa^{2}}{f^{2}-1} \\
& \bar{f}^{3}=1-f^{-3},
\end{aligned}
$$

where $\delta \not \equiv \pm 1$ is an algebraic function of degree 2 at most and $\kappa^{2} \neq 0,1$ is a constant.

Below we will show that Eqs. (3.18)-(3.22) can indeed have meromorphic solutions of finite order.

Let's look at the second-degree Fermat difference Eqs. (3.18), (3.19), and (3.20) first. For Eq. (3.18), we know from [30, Theorem 2] that the solution $f$ is represented as $f=\left(\beta+\beta^{-1}\right) / 2$, where $\beta$ satisfies $\bar{\beta}=i \beta^{ \pm 1}$. For Eq. (3.19), if we put $f=$ $\left(\gamma+\gamma^{-1}\right) / 2$, then we have

$$
\frac{1}{4}\left(\bar{\gamma}-\frac{1}{\bar{\gamma}}\right)^{2}=-\left(\frac{\delta f-1}{f-\delta}\right)^{2}
$$

It follows that

$$
\bar{\gamma}^{2}-2 i \frac{\delta \gamma^{2}-2 \gamma+\delta}{\gamma^{2}-2 \delta \gamma+1} \bar{\gamma}-1=0 .
$$

Solving the above equation, we get the difference Riccati equation

$$
\bar{\gamma}=\left\{-\theta \frac{\left(i \delta-\sqrt{1-\delta^{2}}\right) \gamma+i}{\gamma-\delta+i \sqrt{1-\delta^{2}}}\right\}^{\theta}, \quad \theta= \pm 1
$$

For Eq. (3.20), we have

$$
\bar{f}^{2}=-\frac{8(f+1)}{(f-1)^{2}}
$$

If we put

$$
\sqrt{-8} u=\frac{\bar{f}(f-1)}{f+1}, \quad v=\frac{1}{f+1}
$$


then we have

$$
u^{2}=v, \quad f=\frac{1}{v}-1, \quad \bar{f}=\frac{\sqrt{-8} u}{1-2 v},
$$

and further

$$
(\sqrt{2} \bar{u})^{2}=2 \bar{v}=\frac{2}{\bar{f}+1}=\frac{2\left(2 u^{2}-1\right)}{2 u^{2}-\sqrt{-8} u-1}=1-\left(\frac{\sqrt{2} i u-1}{\sqrt{2} u-i}\right)^{2}
$$

Putting $\sqrt{2} u=\left(\lambda+\lambda^{-1}\right) / 2$, then we have

$$
f=\frac{1-u^{2}}{u^{2}}=\frac{8 \lambda^{2}-\left(\lambda^{2}+1\right)^{2}}{\left(\lambda^{2}+1\right)^{2}}, \quad \bar{\lambda}=\left\{-\theta \frac{-(1+\sqrt{2}) \lambda+i}{\lambda-i+i \sqrt{2}}\right\}^{\theta}, \quad \theta= \pm 1
$$

Since the autonomous version of the difference Riccati equation is solvable explicitly in terms of exponential functions, so are Eqs. (3.18), (3.19), and (3.20) when $\delta$ is a constant.

Equation (3.21) can be rewritten as $\bar{f}^{2} f^{2}-\left(\bar{f}^{2}+f^{2}\right)+\kappa^{2}=0$, which is a special case of symmetric QRT map [17,18]. By doing a suitable Möbius transformation $[4,19]$, for example, $f \rightarrow a(f+1) /(f-1)$, where $a(|a|>1)$ is a constant satisfying $2 a^{4}-2 a^{2}-1=0$ and $\kappa^{2}=a^{4}$, we get

$$
\bar{f}^{2} f^{2}+\bar{f}^{2}+f^{2}+4\left(1+4 a^{2}\right) \bar{f} f+1=0,
$$

which is solvable in terms of Jacobi elliptic functions with finite order of growth [8].

For the third-degree Fermat difference equation (3.22), the solution $f$ satisfies $f^{-1}=H(\varphi), \bar{f}=\eta G(\varphi)$, where $H, G$ are defined in (3.15) and $\varphi$ is an entire function. Choose $\eta=1$. It follows that

$$
\frac{1+\wp^{\prime}(\bar{\varphi}) / \sqrt{3}}{2 \wp(\bar{\varphi})} \cdot \frac{1-\wp^{\prime}(\varphi) / \sqrt{3}}{2 \wp(\varphi)}=1 .
$$

Using the addition law of the Weierstrass elliptic function together with the relation $\wp^{\prime 2}=4 \wp^{3}-1$, it can be shown that this equation is solved by a polynomial of degree 1 satisfying $\bar{\varphi}=\varphi+a$, where $a$ is a constant such that $\wp^{\prime}(a)=-\sqrt{3}$ and $\wp(a)=1$. It follows that the order of growth of $f$ is 2 .

The following three sections contain the proof of Theorem 2. In Sect. 4, we will first find some restrictions on the roots and degrees of the numerator and denominator of $R(z, f)$. This allows us to only consider two cases of Eq. (3.2) where $p=n, q=0$ or $p=q=n$ after a possible bilinear transformation to $f$. These two cases will be discussed in Sects. 5 and 6, respectively. The results obtained in the case $p=n, q=0$ in Sect. 5 are the admissible counterparts of the results on (3.1) by Yanagihara [30]. 


\section{Restrictions on the Roots and Degrees of (3.2)}

We define

$$
R(z, f)=\frac{P(z, f)}{Q(z, f)},
$$

where

$$
P(z, f)=a_{p} f^{p}+a_{p-1} f^{p-1}+\cdots+a_{0}
$$

and

$$
Q(z, f)=b_{q} f^{q}+b_{q-1} f^{q-1}+\cdots+b_{0}
$$

are polynomials in $f$ having no common factors and $p, q \in \mathbb{N}$. Then we have $\operatorname{deg}_{f}(P(z, f))=p, \operatorname{deg}_{f}(Q(z, f))=q$, and by assumption $\operatorname{deg}_{f}(R(z, f))=$ $\max \{p, q\}=n$. In what follows we also write

$$
P(z, f)=a_{p}\left(f-\alpha_{1}\right)^{k_{1}} \cdots\left(f-\alpha_{\mu}\right)^{k_{\mu}}
$$

and

$$
Q(z, f)=b_{q}\left(f-\beta_{1}\right)^{l_{1}} \cdots\left(f-\beta_{\nu}\right)^{l_{\nu}},
$$

where the coefficients $\alpha_{1}, \ldots, \alpha_{\mu}$ and $\beta_{1}, \ldots, \beta_{v}$ are in general algebroid functions and $k_{i}$ and $l_{j}$ denote the order of the roots $\alpha_{i}$ and $\beta_{j}$, respectively. Without losing generality, we may suppose that the greatest common divisor of $k_{1}, \ldots, k_{\mu}, l_{1}, \ldots, l_{\mu}$, which is denoted by $k=\left(k_{1}, \ldots, k_{\mu}, l_{1}, \ldots, l_{\mu}\right)$, is 1 . Otherwise, after taking the $k$-th root on both sides of (3.2), Eq. (3.2) reduces to a difference equation of degree $n / k$ with meromorphic coefficients. Note that under this assumption if $n \geq 2$, then either $P(z, f)$ or $Q(z, f)$ has at least two distinct roots when $p=0$ or $q=0$ or $p=q=n$.

Let now $f$ be an admissible meromorphic solution of (3.2). For the simple case $n=1$, (3.2) is the linear difference equation (3.3) or the difference Riccati equation (3.4). From now on, we take $n$ to be $\geq 2$. By making use of the factorizations (4.1) and (4.2), it follows that the roots of $P(z, f)$ and $Q(z, f)$ are $\alpha_{1}, \ldots, \alpha_{\mu}$ and $\beta_{1}, \ldots, \beta_{v}$, respectively. Suppose that $z_{0} \in \mathbb{C}$ is such that

$$
f\left(z_{0}\right)-\alpha_{i}\left(z_{0}\right)=0
$$

with multiplicity $m \in \mathbb{Z}^{+}$. Now, $n \mid m k_{i}$ since otherwise $z_{0}+1$ would be an algebraic branch point of $f$. Hence, $n \leq m k_{i}$ and so $m \geq n / k_{i}$. The same inequality holds for the roots $\beta_{j}$, as well. Therefore,

$$
\bar{N}\left(r, \frac{1}{f-\alpha_{i}}\right) \leq \frac{k_{i}}{n} N\left(r, \frac{1}{f-\alpha_{i}}\right)
$$

for all $i=1, \ldots, \mu$ and

$$
\bar{N}\left(r, \frac{1}{f-\beta_{j}}\right) \leq \frac{l_{j}}{n} N\left(r, \frac{1}{f-\beta_{j}}\right)
$$


for all $i=1, \ldots, v$. This is trivially true if there is no such $z_{0}$. Note that the above two inequalities (4.3) and (4.4) still hold true for $\bar{f}-\bar{\alpha}_{i}$ and $\bar{f}-\bar{\beta}_{j}$, respectively. Now,

$$
k_{1}+\cdots+k_{\mu} \leq n, \quad l_{1}+\cdots+l_{v} \leq n
$$

and so by applying the Second Main Theorem (2.3) together with (4.3) and (4.4), we have, for any $\varepsilon>0$,

$$
\begin{aligned}
(\mu+v-2-\varepsilon) T(r, f) & \leq \sum_{i=1}^{\mu} \bar{N}\left(r, \frac{1}{f-\alpha_{i}}\right)+\sum_{j=1}^{v} \bar{N}\left(r, \frac{1}{f-\beta_{j}}\right) \\
& \leq \sum_{i=1}^{\mu} \frac{k_{i}}{n} N\left(r, \frac{1}{f-\alpha_{i}}\right)+\sum_{j=1}^{v} \frac{l_{j}}{n} N\left(r, \frac{1}{f-\beta_{j}}\right) \\
& \leq \frac{1}{n}\left(\sum_{i=1}^{\mu} k_{i}+\sum_{j=1}^{v} l_{j}\right) T(r, f) \\
& \leq 2 T(r, f),
\end{aligned}
$$

which implies that $\mu+v \leq 4$ and therefore the combined number of distinct roots of $P(z, f)$ and $Q(z, f)$ is at most 4. In particular, if $k_{1}+\cdots+k_{\mu}<n$ or $l_{1}+\cdots+l_{v}<n$, then $\mu+v \leq 3$.

Consider first the case $p=n$ and $1 \leq q \leq n-1$. We show that this case cannot occur. Suppose that $N(r, f) \neq S(r, f)$. Then there are more than $S(r, f)$ points $z_{0} \in \mathbb{C}$ such that

$$
f(z)=C\left(z-z_{0}\right)^{-m}+O\left(\left(z-z_{0}\right)^{-m+1}\right), \quad C \neq 0, \quad m \in \mathbb{Z}^{+},
$$

in a neighborhood of $z_{0}$. Let these poles be our starting points for iteration. Note that $T(r, f(z+j))=T(r, f)+S(r, f), j \in \mathbb{N}$, and all the coefficients of (3.2) are in $\mathcal{S}^{\prime}(f)$. It follows by [7, Lemma 3.1] that, for an arbitrarily small $\varepsilon \geq 0$, there are more than $S(r, f)$ points $z_{0} \in \mathbb{C}$ at which $f(z+1)^{n}$ has a pole of order at most $(1+\varepsilon)(n-q) m$ and so there are more than $S(r, f)$ poles of $f$ of order at most $(1+\varepsilon)(n-q) m / n$ at $z=z_{0}+1$. By continuing the above iteration it follows that there are more than $S(r, f)$ poles of $f$ of order at most $(1+\varepsilon)^{2}(n-q)^{2} m / n^{2}$ at $z=z_{0}+2$, and more than $S(r, f)$ poles of $f$ of order at most $(1+\varepsilon)^{s}(n-q)^{s} m / n^{s}$ at $z=z_{0}+s, s \in \mathbb{N}$. By letting $s \rightarrow \infty$, it follows by induction that there is necessarily a branch point of $f$ at $z_{0}+s_{0}$ for some $s_{0} \in \mathbb{N}$, a contradiction to our assumption that $f$ is meromorphic. Therefore, we have $N(r, f)=S(r, f)$. Also, from (3.2) we see that $N\left(r, 1 /\left(f-\beta_{j}\right)\right)=S(r, f)$. Thus $\infty, \beta_{j}, j=1, \ldots, v$, are all Picard exceptional small functions of $f$. By the First Main Theorem, we conclude from (4.3) and (2.4) that $\mu=v=1$. It follows that (3.2) assumes the following form:

$$
\bar{f}^{n}=\frac{c\left(f-\alpha_{1}\right)^{n}}{\left(f-\beta_{1}\right)^{q}},
$$


where $\alpha_{1}, \beta_{1}$ are meromorphic functions. Moreover, $\beta_{1} \not \equiv 0$ since otherwise $\alpha_{1}$ is also a Picard exceptional small function of $f$, which is impossible. Set $c_{1}=c^{1 / n}$ and $g=\left(f-\beta_{1}\right)^{1 / n}$. Then $c^{1 / n}, g$ are both algebroid functions of degree at most $n$, and from the above reasoning we have $N(r, g)=S(r, f)$ and $N(r, 1 / g)=S(r, f)$. We take the $n$-th root on both sides of (4.5) and then rewrite the resulting equation as follows:

$$
\bar{g}^{n} g^{q}=c_{1} g^{n}-\bar{\beta}_{1} g^{q}+c_{1}\left(\beta_{1}-\alpha_{1}\right) .
$$

Let $u$ be a function such that

$$
c_{1} u^{n}-\bar{\beta}_{1} u^{q}+c_{1}\left(\beta_{1}-\alpha_{1}\right)=0 .
$$

Then $u$ is an algebroid function of degree at most $n^{2}$. Since $q \leq n-1$ and $\beta_{1} \not \equiv$ $\alpha_{1}$, the above equation has at least one nonzero root $u_{0}$ and from (4.6) we see that $N\left(r, 1 /\left(g-u_{0}\right)\right)=S(r, g)$. Since $T(r, f)=n T(r, g)+S(r, f)$, then $u_{0}^{n}+\beta_{1}$ is also a Picard exceptional small function of $f$, which is impossible. Therefore, the case where $p=n$ and $1 \leq q \leq n-1$ cannot occur. This also implies that the case where 0 is a root of $P(z, f)$ of order less than $n$ cannot occur since otherwise by doing a bilinear transformation $f \rightarrow 1 / f$ to (3.2), we get

$$
\bar{f}^{n}=\frac{P_{1}(z, f)}{Q_{1}(z, f)},
$$

where $P_{1}(z, f)$ is a polynomial in $f$ of degree $n$ and $Q_{1}(z, f)$ is a polynomial in $f$ of degree less than $n$, which is impossible. From the above reasoning, we conclude that $q=0$ or $q=n$ when $p=n$ and that 0 is not a root of $P(z, f)$ of order less than $n$.

Consider now the case $q=n$ and $0 \leq p \leq n-1$. Below we show that in this case 0 cannot be a root of $Q(z, f)$ of order less than $n$. Otherwise, (3.2) can be written as

$$
\bar{f}^{n}=\frac{P(z, f)}{f^{l_{0}} \hat{Q}(z, f)},
$$

where $1 \leq l_{0} \leq n-1$, and $\hat{Q}(z, f)$ is a polynomial in $f$ of degree $n-l_{0}$. If $N(r, 1 / f) \neq S(r, f)$, then there are more than $S(r, f)$ points $z_{0} \in \mathbb{C}$ such that

$$
f(z)=C\left(z-z_{0}\right)^{m}+O\left(\left(z-z_{0}\right)^{m+1}\right), \quad C \neq 0, \quad m \in \mathbb{N}^{+}
$$

in a neighborhood of $z_{0}$. We now iterate (3.2) with these points as our starting points. Recall that $T(r, f(z+j))=T(r, f)+S(r, f)$ for all $j \in \mathbb{N}$ and all the coefficients of (3.2) are in $\mathcal{S}^{\prime}(f)$. According to [7, Lemma 3.1], for an arbitrarily small $\varepsilon \geq 0$, by (4.7), there are more than $S(r, f)$ points $z_{0} \in \mathbb{C}$ at which $f(z+1)^{n}$ has a pole of order at most $(1+\varepsilon) l_{0} m$ and so there are more than $S(r, f)$ poles of $f$ of order at most $(1+\varepsilon) l_{0} m / n$ at $z=z_{0}+1$. It follows that there are more than $S(r, f)$ points $z_{0} \in \mathbb{C}$ at which $f(z+2)^{n}$ has a zero of order at most $(1+\varepsilon) l_{0}(n-p) m$, that is, there are more than $S(r, f)$ zeros of $f$ of order at most $(1+\varepsilon) l_{0}(n-p) m / n$ at $z=z_{0}+2$. Then, by continuing the iteration it follows that there are more than $S(r, f)$ zeros 
of $f$ of order $(1+\varepsilon)^{s}(n-p)^{s} l_{0}^{s} m / n^{s}$ at $z=z_{0}+2 s, s \in \mathbb{N}$. By letting $s \rightarrow \infty$, it follows by induction that there is necessarily a branch point of $f$ at $z_{0}+2 s_{0}$ for some $s_{0} \in \mathbb{N}$, a contradiction to our assumption that $f$ is meromorphic. Therefore, $N(r, 1 / f)=S(r, f)$. Also, from (4.7) we see that $N(r, f)=S(r, f)$ since $p<q$ and then, since $l_{0} \leq n-1$, it follows that there exists another nonzero $\beta_{j}$ such that $N\left(r, 1 /\left(f-\beta_{j}\right)=S(r, f)\right.$, that is to say, $f$ has at least 3 Picard exceptional small functions, which is impossible. Therefore, 0 cannot be a root of $Q(z, f)$ of order $<n$ when $0 \leq p \leq n-1$. Now, since 0 is not a root of $P(z, f)$ either, by doing a bilinear transformation $f \rightarrow 1 / f$ to (3.2), we get

$$
\bar{f}^{n}=\frac{P_{2}(z, f)}{Q_{2}(z, f)}
$$

where $P_{2}(z, f)$ and $Q_{2}(z, f)$ are two polynomials in $f$, both of them having degree equal to $n$. In particular, $Q_{2}(z, f)=f^{n}$ when $p=0$.

From the above discussions, we conclude that we only need to consider (3.2) for two cases where $p=n, q=0$, or $p=q=n$. Moreover, if $P(z, f)$ has two or more distinct roots, then none of them vanishes identically.

\section{Equation (3.2) with $p=n$ and $q=0$}

In Sect. 4, we have shown that the combined number of distinct roots of $P(z, f)$ is at most 3 when $p=n$ and $q=0$ and assuming that $P(z, f)$ has at least 2 distinct roots. Hence we have the following two possibilities:

$$
\begin{aligned}
& \bar{f}^{n}=c\left(f-\alpha_{1}\right)^{\kappa_{1}}\left(f-\alpha_{2}\right)^{\kappa_{2}}, \quad \alpha_{1} \alpha_{2} \not \equiv 0, \\
& \bar{f}^{n}=c\left(f-\alpha_{1}\right)^{\mu_{1}}\left(f-\alpha_{2}\right)^{\mu_{2}}\left(f-\alpha_{3}\right)^{\mu_{3}}, \quad \alpha_{1} \alpha_{2} \alpha_{3} \not \equiv 0,
\end{aligned}
$$

where $\kappa_{i}, \mu_{j}$ are positive integers satisfying $\kappa_{1}+\kappa_{2}=n, \mu_{1}+\mu_{2}+\mu_{3}=n$.

We first look at Eq. (5.1). Note that $\alpha_{1}, \alpha_{2}$ are both meromorphic functions when $n \geq 3$ and are algebroid functions of degree at most 2 when $n=2$. Suppose that $\kappa_{1}+\kappa_{2}=n \geq 3$. Since $\left(\kappa_{1}, \kappa_{2}\right)=\left(n, \kappa_{2}\right)=\left(n, \kappa_{2}\right)=1$, from $(5.1)$ we see that the multiplicity of zero $z_{0}$ of $f-\alpha_{1}$ (also of $f-\alpha_{2}$ ) is at least $n$ since otherwise $z_{0}$ would be an algebraic branch point of $f$. Thus we have

$$
\bar{N}\left(r, \frac{1}{f-\alpha_{i}}\right) \leq \frac{1}{n} N\left(r, \frac{1}{f-\alpha_{i}}\right), \quad i=1,2 .
$$

If $n=3$ or $n=4$, then obviously $\min \left\{\kappa_{1}, \kappa_{2}\right\}=1$; if $n \geq 5$ and $\min \left\{\kappa_{1}, \kappa_{2}\right\} \geq 2$, then from (5.1) we see that $f$ is also completely ramified at zeros, which, with the First Main Theorem and (2.4), yields that $2-2 / n+1 / 2 \leq 2$, a contradiction. Hence we must have $\min \left\{\kappa_{1}, \kappa_{2}\right\}=1$, and it follows that (5.1) is of the form

$$
\bar{f}^{n}=c\left(f-\alpha_{1}\right)^{n-1}\left(f-\alpha_{2}\right)
$$


Moreover, since $n \geq 3$, we have $N\left(r, 1 /\left(f-\alpha_{2}\right)\right) \neq S(r, f)$, for otherwise $f$ is $n$-fold ramified at $\alpha_{1}$-points and $(n-1)$-fold ramified at zeros, which yields a contradiction to (2.4). Write $c_{1}=\left[c\left(\alpha_{2}-\alpha_{1}\right)\right]^{1 / n}$ and $c_{2}=1 /\left(\alpha_{2}-\alpha_{1}\right)$. Then $c_{1}$ is an algebroid function of degree at most $n$. Put

$$
c_{1} u=\frac{\bar{f}}{f-\alpha_{1}}, \quad v=\frac{1}{f-\alpha_{1}},
$$

where $u$ is an algebroid function with at most $S(r, f)$ many branch points. By the Valiron-Mohon'ko identity [15,26] (see also [13]) we have $T(r, v)=T(r, f)+$ $S(r, f)$, and thus all the coefficients of (5.3) are small functions of $v$. Then we have

$$
f=\frac{1}{v}+\alpha_{1}, \quad \bar{f}=\frac{c_{1} u}{v},
$$

and from (5.3) and (5.4) that

$$
u^{n}=c_{2}-v
$$

It also follows that

$$
\frac{c_{1} u-\bar{\alpha}_{2} v}{v}=\bar{f}-\bar{\alpha}_{2} .
$$

Since zeros of $\bar{f}-\bar{\alpha}_{2}$ are $n$-fold ramified, we see that zeros of $F=c_{1} u-\bar{\alpha}_{2} v$ are also $n$-fold ramified. Let $u_{0}$ and $v_{0}$ be two functions such that

$$
u_{0}^{n}=c_{2}-v_{0}, \quad c_{1} u_{0}-\bar{\alpha}_{2} v_{0}=0 .
$$

Eliminating $u_{0}$ from the above two equations gives

$$
\bar{\alpha}_{2}^{n} v_{0}^{n}+c_{1}^{n} v_{0}-c_{1}^{n} c_{2}=0
$$

from which we see that $v_{0}$ is an algebroid function of degree at most $n$. Moreover, it is easy to see that $c_{2}$ is not a root of (5.7). If (5.7) has only one root, then, since $n \geq 3$, by Vieta's formulas we see that $v_{0}=0$ and it follows from (5.7) that $c_{1}^{n} c_{2} \equiv 0$, a contradiction. Therefore, (5.7) has at least two distinct roots, say, $v_{1}$ and $v_{2}$. Rewrite (5.6) as

$$
-\frac{\bar{\alpha}_{2}^{n} v^{n}+c_{1}^{n} v-c_{1}^{n} c_{2}}{v^{n}}=\bar{f}^{n}-\bar{\alpha}_{2}^{n} .
$$

Denote the order of the roots $v_{1}$ and $v_{2}$ by $l_{1}$ and $l_{2}$, respectively. Then $1 \leq l_{1}, l_{2} \leq$ $n-1$, and we have

$$
\bar{\alpha}_{2}^{n} v^{n}+c_{1}^{n} v-c_{1}^{n} c_{2}=\bar{\alpha}_{2}^{n}\left(v-v_{1}\right)^{l_{1}}\left(v-v_{2}\right)^{l_{2}} P(z, v)
$$

where $P(z, v)$ is a polynomial in $v$ of degree $n-l_{1}-l_{2}$ with small functions of $v$ as coefficients. Suppose that $z_{0} \in \mathbb{C}$ is such that

$$
f\left(z_{0}+1\right)-\alpha_{2}\left(z_{0}+1\right)=0
$$


with multiplicity $n_{0} \geq n$. If $v\left(z_{0}\right)-v_{1}\left(z_{0}\right)=0$ with multiplicity $m \in \mathbb{Z}^{+}$, then from (5.8), we have $n_{0} \mid m l_{1}$. Hence, $n_{0} \leq m l_{1}$ and so $m \geq 2$. Therefore,

$$
\bar{N}\left(r, \frac{1}{v-v_{1}}\right) \leq \frac{1}{2} N\left(r, \frac{1}{v-v_{1}}\right) .
$$

The same inequality holds for $v_{2}$, as well. Thus $v$ is completely ramified at $v_{1}$-points and $v_{2}$-points. But from (5.4) we see that $v$ is $n$-fold ramified at poles and $c_{2}$-points, which yields a contradiction to (2.4) since $n \geq 3$. Hence $n=2$ and we therefore have

$$
\bar{f}^{2}=c\left(f-\alpha_{1}\right)\left(f-\alpha_{2}\right)
$$

In what follows, we will still use the expressions in (5.4) to transform (5.9) into (5.5) for the case $n=2$. Moreover, from the above reasoning we know that

$$
\bar{\alpha}_{2}^{2} v_{0}^{2}+c_{1}^{2} v_{0}-c_{1}^{2} c_{2}=0
$$

which has two distinct roots if

$$
\left(4 \bar{\alpha}_{2}^{2} c_{2}+c_{1}^{2}\right) c_{1}^{2} \neq 0
$$

If (5.10) holds, then $v$ must be completely ramified at poles, $c_{2}$-points, $v_{1}$-points, and $v_{2}$-points; if not, then we have

$$
c_{1}^{2}+4 \bar{\alpha}_{2}^{2} c_{2}=\frac{4 \bar{\alpha}_{2}^{2}+c\left(\alpha_{2}-\alpha_{1}\right)^{2}}{\alpha_{2}-\alpha_{1}}=0 .
$$

Rewrite (5.9) as

$$
\bar{f}^{2}=c\left(g^{2}-c_{4}^{2}\right),
$$

where $g=f-c_{3}, c_{3}=\left(\alpha_{1}+\alpha_{2}\right) / 2$ are both meromorphic functions and $c_{4}=$ $\left(\alpha_{2}-\alpha_{1}\right) / 2=1 /\left(2 c_{2}\right)$ is an algebroid function of degree at most 2 . Note that $c_{4}^{2}=$ $\left[\left(\alpha_{2}+\alpha_{1}\right)^{2}-4 \alpha_{1} \alpha_{2}\right] / 4$ is a meromorphic function. Put

$$
g=\frac{c_{4}}{2}\left(\beta+\frac{1}{\beta}\right) \text {. }
$$

Then substitution yields

$$
\bar{f}^{2}=\frac{c c_{4}^{2}}{4}\left(\beta-\frac{1}{\beta}\right)^{2},
$$

and, without loss of generality, we may suppose that

$$
\beta^{2}-\frac{2}{c^{1 / 2} c_{4}}\left(\bar{\alpha}_{1}+\frac{1}{\bar{v}}\right) \beta-1=0 .
$$


Note that $4 c_{2}^{2} c_{4}^{2}=1$ and $c=c_{1}^{2} c_{2}$. We have $4 c c_{4}^{2}=c_{1}^{2} / c_{2}$. The discriminant of the quadratic equation (5.12) with respect to $\beta$,

$$
\frac{4\left(\bar{\alpha}_{1} \bar{v}+1\right)^{2}+4 c c_{4}^{2} \bar{v}^{2}}{c c_{4}^{2} \bar{v}^{2}}=\frac{4 c_{2}\left(\bar{\alpha}_{1} \bar{v}+1\right)^{2}+c_{1}^{2} \bar{v}^{2}}{c_{2} c c_{4}^{2} \bar{v}^{2}}=\frac{4 \bar{f}^{2}}{c c_{4}^{2}}+4=\frac{4\left(c c_{4}^{2}+\bar{f}^{2}\right)}{c c_{4}^{2}}=\frac{4 g^{2}}{c_{4}^{2}}
$$

implies that $\beta$ is meromorphic apart from at most $S(r, f)$ many points. Moreover, roots of the equation

$$
\left(4 \bar{\alpha}_{1}^{2} c_{2}+c_{1}^{2}\right) \bar{v}^{2}+8 c_{2} \bar{\alpha}_{1} \bar{v}+4 c_{2}=0
$$

with respect to $\bar{v}$ must be either $c_{2}$ or coincide with $v_{1}, v_{2}$.

If $c_{2}$ is a root of (5.13), then we have

$$
\frac{4 \bar{\alpha}_{1}^{2} c_{2}+c_{1}^{2}}{\left(\bar{\alpha}_{2}-\bar{\alpha}_{1}\right)^{2}}+\frac{8 c_{2} \bar{\alpha}_{1}}{\bar{\alpha}_{2}-\bar{\alpha}_{1}}+4 c_{2}=0
$$

which yields (5.11). On the other hand, by exchanging the roles of $\alpha_{1}$ and $\alpha_{2}$, we also have

$$
c_{1}^{2}+4 \bar{\alpha}_{1}^{2} c_{2}=0
$$

From (5.11) and (5.14) we see that $\alpha_{2}=-\alpha_{1}$, and it follows that $c=-\bar{\alpha}_{1}^{2} / \alpha_{1}^{2}$. By doing the transformation $f \rightarrow \alpha_{1} f$, (5.9) becomes

$$
\bar{f}^{2}=1-f^{2}
$$

which is the Eq. (3.5).

If $c_{2}$ is not a root of (5.13), then roots of (5.13) must coincide with $v_{1}, v_{2}$, which means that

$$
\frac{\overline{\bar{\alpha}}_{2}^{2}}{4 \bar{\alpha}_{1}^{2} c_{2}+c_{1}^{2}}=\frac{\bar{c}_{1}^{2}}{8 c_{2} \bar{\alpha}_{1}}=\frac{-\bar{c}_{1}^{2} \bar{c}_{2}}{4 c_{2}} .
$$

Recall that $c_{1}=\left[c\left(\alpha_{2}-\alpha_{1}\right)\right]^{1 / 2}$ and $c_{2}=1 /\left(\alpha_{2}-\alpha_{1}\right)$. The second equation of (5.15) gives $-2 \bar{c}_{2} \bar{\alpha}_{1}=1$, i.e., $\bar{\alpha}_{2}=-\bar{\alpha}_{1}$. It follows from the first equation of (5.15) that

$$
\alpha_{1}^{2} \bar{c} c+\bar{\alpha}_{1}^{2} \bar{c}+\overline{\bar{\alpha}}_{1}^{2}=0
$$

Set $d=c \alpha_{1}^{2} / \bar{\alpha}_{1}^{2}$. Then $d$ is a meromorphic function satisfying $\bar{d}(d+1)+1=0$. By doing the transformation $f \rightarrow \alpha_{1} f$, (5.9) becomes

$$
\bar{f}^{2}=d\left(f^{2}-1\right)
$$

which is the Eq. (3.6).

We now look at Eq. (5.2). Set $k_{1}=\left(n, \mu_{1}\right), k_{2}=\left(n, \mu_{2}\right), k_{3}=\left(n, \mu_{3}\right)$, and let $K_{1}=n / k_{1}, K_{2}=n / k_{2}, K_{3}=n / k_{3}$. Obviously, $K_{i} \geq 2, i=1,2,3$, and 
$1 / K_{1}+1 / K_{2}+1 / K_{3}=\left(k_{1}+k_{2}+k_{3}\right) / n \leq 1$. Now $f$ is $K_{i}$-fold ramified at $\alpha_{i}$-points, $i=1,2,3$, which with (2.4) implies that

$$
1-\frac{1}{K_{1}}+1-\frac{1}{K_{2}}+1-\frac{1}{K_{3}} \leq 2
$$

and so $1 / K_{1}+1 / K_{2}+1 / K_{3}=\left(k_{1}+k_{2}+k_{3}\right) / n=1$. Thus we have the following three possibilities:

$$
\begin{array}{ll}
1, & K_{1}=2, K_{2}=3, K_{3}=6 ; \\
2, & K_{1}=2, K_{2}=4, K_{3}=4 ; \\
3, & K_{1}=3, K_{2}=3, K_{3}=3 ;
\end{array}
$$

which implies that (5.2) assumes one of the following three forms:

$$
\begin{aligned}
& \bar{f}^{3}=c\left(f-\alpha_{1}\right)\left(f-\alpha_{2}\right)\left(f-\alpha_{3}\right), \quad \alpha_{1} \alpha_{2} \alpha_{3} \not \equiv 0, \\
& \bar{f}^{4}=c\left(f-\alpha_{1}\right)^{2}\left(f-\alpha_{2}\right)\left(f-\alpha_{3}\right), \quad \alpha_{1} \alpha_{2} \alpha_{3} \not \equiv 0, \\
& \bar{f}^{6}=c\left(f-\alpha_{1}\right)^{3}\left(f-\alpha_{2}\right)^{2}\left(f-\alpha_{3}\right), \quad \alpha_{1} \alpha_{2} \alpha_{3} \not \equiv 0 .
\end{aligned}
$$

Note that $\alpha_{1}$ in (5.17) and $\alpha_{1}, \alpha_{2}, \alpha_{3}$ in (5.18) are all meromorphic functions.

Let's first consider Eq. (5.16). Put

$$
\frac{\bar{f}}{f-\alpha_{1}}=c_{1} u, \quad \frac{1}{f-\alpha_{1}}=b_{2} v+b_{1},
$$

where $u$ and $v$ are two algebroid functions with at most $S(r, f)$ many branch points and

$$
\begin{aligned}
& c_{1}=\left[4 c b_{2}^{2}\left(\alpha_{2}-\alpha_{1}\right)\left(\alpha_{3}-\alpha_{1}\right)\right]^{1 / 3}, \\
& b_{1}=\frac{1}{2}\left(\frac{1}{\alpha_{2}-\alpha_{1}}+\frac{1}{\alpha_{3}-\alpha_{1}}\right), \\
& b_{2}=\frac{1}{2}\left(\frac{1}{\alpha_{2}-\alpha_{1}}-\frac{1}{\alpha_{3}-\alpha_{1}}\right)
\end{aligned}
$$

are algebroid functions of degrees at most 9, 3, 3, respectively. By writing $v$ in terms of $f$ with small coefficients and the Valiron-Mohon'ko identity [15,26] (see also [13]), we have $T(r, v)=T(r, f)+S(r, f)$ and thus all the coefficients of (5.16) are small functions of $v$. Then we have

$$
f=\alpha_{1}+\frac{1}{b_{2} v+b_{1}}, \quad \bar{f}=\frac{c_{1} u}{b_{2} v+b_{1}},
$$

and from (5.16) and (5.19) that

$$
v^{2}=4 u^{3}+1
$$

It also follows that

$$
\frac{c_{1} u-\bar{\alpha}_{2}\left(b_{2} v+b_{1}\right)}{b_{2} v+b_{1}}=\bar{f}-\bar{\alpha}_{2} .
$$


Since zeros of $\bar{f}-\bar{\alpha}_{2}$ are threefold ramified, we see that zeros of $F=c_{1} u-\bar{\alpha}_{2}\left(b_{2} v+\right.$ $\left.b_{1}\right)$ are also threefold ramified. Let $u_{0}$ and $v_{0}$ be two functions such that

$$
v_{0}^{2}=4 u_{0}^{3}+1, \quad c_{1} u_{0}-\bar{\alpha}_{2}\left(b_{2} v_{0}+b_{1}\right)=0 .
$$

Eliminating $u_{0}$ from the above two equations gives

$$
4 \bar{\alpha}_{2}^{3}\left(b_{2} v_{0}+b_{1}\right)^{3}-c_{1}^{3}\left(v_{0}^{2}-1\right)=0,
$$

from which we see that $v_{0}$ is an algebroid function of degree at most 27 . Moreover, it is seen that \pm 1 are not roots of (5.23), for otherwise we have $b_{2} \pm b_{1}=0$, a contradiction. Rewrite (5.22) as

$$
-\frac{4 \bar{\alpha}_{2}^{3}\left(b_{2} v+b_{1}\right)^{3}-c_{1}^{3}\left(v^{2}-1\right)}{4\left(b_{2} v+b_{1}\right)^{3}}=\bar{f}^{3}-\bar{\alpha}_{2}^{3} .
$$

From Eq. (5.21), we see that $v$ is threefold ramified over $\pm 1, \infty$. This implies that (5.23) can admit only one root, for otherwise by a similar analysis as to (5.8) we obtain from (5.24) that (5.23) has at least two roots, say, $v_{1}$ and $v_{2}$, distinct from $\pm 1, \infty$ such that $v$ is completely ramified at $v_{1}$-points and $v_{2}$-points, which is impossible by Theorem 1. Expanding (5.23) gives

$$
4 \bar{\alpha}_{2}^{3} b_{2}^{3} v_{0}^{3}+\left(12 \bar{\alpha}_{2}^{3} b_{2}^{2} b_{1}-c_{1}^{3}\right) v_{0}^{2}+12 \bar{\alpha}_{2}^{3} b_{2} b_{1}^{2} v_{0}+4 \bar{\alpha}_{2}^{3} b_{1}^{3}+c_{1}^{3}=0
$$

and so by Vieta's formulas, we get

$$
\frac{12 \bar{\alpha}_{2}^{3} b_{2}^{2} b_{1}-c_{1}^{3}}{4 \bar{\alpha}_{2}^{3} b_{2}^{3}}=-3 v_{0}, \quad \frac{3 b_{1}^{2}}{b_{2}^{2}}=3 v_{0}^{2} .
$$

From the second equation of (5.25) we get $v_{0}= \pm b_{1} / b_{2}$. If $v_{0}=-b_{1} / b_{2}$, then substitution into (5.23) yields $b_{1}^{2}=b_{2}^{2}$, a contradiction. Hence $v_{0}=b_{1} / b_{2}$. Combining this equation with the first equation of (5.25), we get

$$
c_{1}^{3}=24 \bar{\alpha}_{2}^{3} b_{2}^{2} b_{1}
$$

Similarly, the above equation also holds when replacing $\bar{\alpha}_{2}$ in (5.22) with $\bar{\alpha}_{1}$ and $\bar{\alpha}_{3}$, which implies that $\alpha_{1}^{3}=\alpha_{2}^{3}=\alpha_{3}^{3}$. Thus, $\alpha_{2}=\eta \alpha_{1}, \alpha_{3}=\eta^{2} \alpha_{1}$, where $\eta$ is the cubic root of 1 satisfying $\eta^{2}+\eta+1=0$. Moreover, we have

$$
c_{1}^{3}=4 c b_{2}^{2}\left(\alpha_{2}-\alpha_{1}\right)\left(\alpha_{3}-\alpha_{1}\right)=24 \bar{\alpha}_{1}^{3} b_{2}^{2} b_{1},
$$

which yields $c=-\bar{\alpha}_{1}^{3} / \alpha_{1}^{3}$. By doing the transformation $f \rightarrow \alpha_{1} f,(5.16)$ becomes

$$
\bar{f}^{3}=1-f^{3}
$$

which is the Eq. (3.13). 
We now consider Eq. (5.17). Put

$$
\frac{\bar{f}}{f-\alpha_{1}}=c_{1} u, \quad \frac{1}{f-\alpha_{1}}=b_{2} v+b_{1},
$$

where $u$ and $v$ are two algebroid functions with at most $S(r, f)$ many branch points and

$$
\begin{aligned}
& c_{1}=\left[-b_{2}^{2} c\left(\alpha_{2}-\alpha_{1}\right)\left(\alpha_{3}-\alpha_{1}\right)\right]^{1 / 4}, \\
& b_{1}=\frac{1}{2}\left(\frac{1}{\alpha_{2}-\alpha_{1}}+\frac{1}{\alpha_{3}-\alpha_{1}}\right), \\
& b_{2}=\frac{1}{2}\left(\frac{1}{\alpha_{2}-\alpha_{1}}-\frac{1}{\alpha_{3}-\alpha_{1}}\right)
\end{aligned}
$$

are algebroid functions of degrees at most 8, 2, 2, respectively. By writing $v$ in terms of $f$ with small coefficients and the Valiron-Mohon'ko identity [15,26] (see also [13]), we have $T(r, v)=T(r, f)+S(r, f)$ and thus all the coefficients of (5.17) are small functions of $v$. Then we have

$$
f=\frac{1}{b_{2} v+b_{1}}+\alpha_{1}, \quad \bar{f}=\frac{c_{1} u}{b_{2} v+b_{1}}
$$

and from (5.17) and (5.26) that

$$
v^{2}=1-u^{4}
$$

It also follows that

$$
\frac{c_{1} u-\bar{\alpha}_{2}\left(b_{2} v+b_{1}\right)}{b_{2} v+b_{1}}=\bar{f}-\bar{\alpha}_{2} .
$$

Since zeros of $\bar{f}-\bar{\alpha}_{2}$ are fourfold ramified, we see that zeros of $F=c_{1} u-\bar{\alpha}_{2}\left(b_{2} v+b_{1}\right)$ are also fourfold ramified. Let $u_{0}$ and $v_{0}$ be two functions such that

$$
v_{0}^{2}=1-u_{0}^{4}, \quad c_{1} u_{0}-\bar{\alpha}_{2} b_{2} v_{0}-\bar{\alpha}_{2} b_{1}=0 .
$$

Eliminating $u_{0}$ from the above two equations gives

$$
\bar{\alpha}_{2}^{4}\left(b_{2} v_{0}+b_{1}\right)^{4}-c_{1}^{4}\left(1-v_{0}^{2}\right)=0
$$

from which we see that $v_{0}$ is an algebroid function of degree at most 16 . Moreover, it is seen that \pm 1 are not roots of (5.30), for otherwise we have $b_{2} \pm b_{1}=0$, a contradiction. Rewrite (5.29) as

$$
\frac{c_{1}^{4}\left(1-v^{2}\right)-\bar{\alpha}_{2}^{4}\left(b_{2} v+b_{1}\right)^{4}}{\left(b_{2} v+b_{1}\right)^{4}}=\bar{f}^{4}-\bar{\alpha}_{2}^{4} .
$$

From Eq. (5.28), we see that $v$ is completely ramified over $\pm 1, \infty$. This implies that (5.30) can admit only one root, for otherwise by a similar analysis as to (5.8) we obtain from (5.31) that (5.30) has at least two roots, say, $v_{1}$ and $v_{2}$, distinct from $\pm 1, \infty$ 
such that $v$ is completely ramified at $v_{1}$-points and $v_{2}$-points, which is impossible by Theorem 1. Expanding (5.30) gives

$$
\bar{\alpha}_{2}^{4}\left(b_{2}^{4} v_{0}^{4}+4 b_{2}^{3} b_{1} v_{0}^{3}+\ldots+b_{1}^{4}\right)-c_{1}^{4}\left(1-v_{0}^{2}\right)=0,
$$

and so by Vieta's formulas, we obtain $4 b_{1} / b_{2}=-4 v_{0}$, which gives $b_{2} v_{0}+b_{1}=0$ and it follows from (5.30) that $v_{0}^{2}=1$, a contradiction. Therefore, (5.17) cannot admit any meromorphic solutions.

Finally, let's consider Eq. (5.18). Put

$$
\frac{\bar{f}}{f-\alpha_{1}}=c_{1} u, \quad \frac{1}{f-\alpha_{1}}=b_{2} v-b_{1},
$$

where $u$ is an algebroid function with at most $S(r, f)$ many branch points and

$$
\begin{aligned}
& c_{1}=\left[-c b_{2}^{3}\left(\alpha_{2}-\alpha_{1}\right)^{2}\left(\alpha_{3}-\alpha_{1}\right)\right]^{1 / 6} \\
& b_{1}=-\frac{1}{3}\left(\frac{2}{\alpha_{2}-\alpha_{1}}+\frac{1}{\alpha_{3}-\alpha_{1}}\right) \\
& b_{2}=\frac{1}{\alpha_{2}-\alpha_{1}}+b_{1}=\frac{1}{3}\left(\frac{1}{\alpha_{2}-\alpha_{1}}-\frac{1}{\alpha_{3}-\alpha_{1}}\right)
\end{aligned}
$$

and $c_{1}$ is an algebroid function of degree at most 6. By writing $v$ in terms of $f$ with small coefficients and the Valiron-Mohon'ko identity [15,26] (see also [13]), we have $T(r, v)=T(r, f)+S(r, f)$, and thus all the coefficients of (5.18) are small functions of $v$. Then we have

$$
f=\frac{1}{b_{2} v-b_{1}}+\alpha_{1}, \quad \bar{f}=\frac{c_{1} u}{b_{2} v-b_{1}}
$$

and from (5.18) and (5.32) that

$$
u^{6}=(v-1)^{2}(v+2)=v^{3}-3 v+2 .
$$

It also follows that

$$
\frac{c_{1} u-\bar{\alpha}_{3}\left(b_{2} v-b_{1}\right)}{b_{2} v-b_{1}}=\bar{f}-\bar{\alpha}_{3} .
$$

Since zeros of $\bar{f}-\bar{\alpha}_{3}$ are sixfold ramified, we see that zeros of $F=c_{1} u-\bar{\alpha}_{3}\left(b_{2} v-b_{1}\right)$ are also sixfold ramified. Let $u_{0}$ and $v_{0}$ be two functions such that

$$
u_{0}^{6}=v_{0}^{3}-3 v_{0}+2, \quad c_{1} u_{0}-\bar{\alpha}_{3} b_{2} v_{0}+\bar{\alpha}_{3} b_{1}=0
$$

Eliminating $u_{0}$ from the above two equations gives

$$
\bar{\alpha}_{2}^{6}\left(b_{2} v_{0}-b_{1}\right)^{6}-c_{1}^{6}\left(v_{0}^{3}-3 v_{0}+2\right)=0,
$$

from which we see that $v_{0}$ is an algebroid function of degree at most 6 . Moreover, it is seen that 1 and -2 are not roots of (5.36), for otherwise we have $b_{2}-b_{1}=0$ or 
$2 b_{2}+b_{1}=0$, a contradiction. Rewrite (5.35) as

$$
\frac{c_{1}^{6}\left(v^{3}-3 v+2\right)-\bar{\alpha}_{3}^{6}\left(b_{2} v-b_{1}\right)^{6}}{\left(b_{2} v-b_{1}\right)^{6}}=\bar{f}^{6}-\bar{\alpha}_{3}^{6} .
$$

From Eq. (5.34), we see that $v$ is completely ramified over $1,-2, \infty$. This implies that (5.36) can admit only one root, for otherwise by a similar analysis as to (5.8) we obtain from (5.37) that (5.36) has at least two roots, say, $v_{1}$ and $v_{2}$, distinct from $1,-2, \infty$ such that $v$ is completely ramified at $v_{1}$-points and $v_{2}$-points, which is impossible by Theorem 1. Expanding (5.36) gives

$$
\bar{\alpha}_{2}^{6}\left(b_{2}^{6} v_{0}^{6}-6 b_{2}^{5} b_{1} v_{0}^{5}+\cdots+b_{1}^{6}\right)-c_{1}^{6}\left(v_{0}^{3}-3 v_{0}+2\right)=0,
$$

and so by Vieta's formulas, we obtain $-6 b_{1} / b_{2}=-6 v_{0}$, which gives $b_{2} v_{0}-b_{1}=0$ and it follows from (5.36) that $v_{0}^{3}-3 v_{0}+2=0$, a contradiction. Therefore, (5.18) cannot admit any meromorphic solutions.

\section{Equation (3.2) with $p=q=n$}

In this section, we consider the cases where $Q(z, f)$ has one, two, or three roots.

\section{1 $Q(z, f)$ has Only One Root}

In Sect. 4, we have shown that the combined number of distinct roots of $P(z, f)$ and $Q(z, f)$ is at most 4 and assumed that $P(z, f)$ has at least 2 distinct roots. Hence we have the following two possibilities:

$$
\begin{aligned}
\bar{f}^{n} & =\frac{c\left(f-\alpha_{1}\right)^{\kappa_{1}}\left(f-\alpha_{2}\right)^{\kappa_{2}}}{\left(f-\beta_{1}\right)^{n}}, \quad \alpha_{1} \alpha_{2} \not \equiv 0, \\
\bar{f}^{n} & =\frac{c\left(f-\alpha_{1}\right)^{\mu_{1}}\left(f-\alpha_{2}\right)^{\mu_{2}}\left(f-\alpha_{3}\right)^{\mu_{3}}}{\left(f-\beta_{1}\right)^{n}}, \quad \alpha_{1} \alpha_{2} \alpha_{3} \not \equiv 0,
\end{aligned}
$$

where $\kappa_{i}, \mu_{j}$ are positive integers satisfying $\kappa_{1}+\kappa_{2}=n, \mu_{1}+\mu_{2}+\mu_{3}=n$.

We now look at Eq. (6.1). Note that $\alpha_{1}, \alpha_{2}$ are both meromorphic functions when $n \geq 3$ and are algebroid functions of degree at most 2 when $n=2$. Suppose $\kappa_{1}+\kappa_{2}=$ $n \geq 3$. By a similar analysis on the $\alpha_{i}$-points of $f$ in (6.1) as on the $\alpha_{i}$-points of $f$ in (5.1) in Sect. 5, we can obtain that (6.1) is of the form

$$
\bar{f}^{n}=\frac{c\left(f-\alpha_{1}\right)^{n-1}\left(f-\alpha_{2}\right)}{\left(f-\beta_{1}\right)^{n}} .
$$


Moreover, we have $N\left(r, 1 /\left(f-\alpha_{2}\right)\right) \neq S(r, f)$. Set $c_{1}=\left[c\left(\alpha_{2}-\alpha_{1}\right)\right]^{1 / n}$ and $c_{2}=1 /\left(\alpha_{2}-\alpha_{1}\right)$. Then $c_{1}$ is an algebroid function of degree at most $n$. Put

$$
c_{1} u=\frac{\bar{f}\left(f-\beta_{1}\right)}{f-\alpha_{1}}, \quad v=\frac{1}{f-\alpha_{1}},
$$

where $u$ is an algebroid function with at most $S(r, f)$ many branch points. Note that all the coefficients of (6.3) are small functions of $v$. Then we have

$$
f=\frac{1}{v}+\alpha_{1}, \quad \bar{f}=\frac{c_{1} u}{1+\left(\alpha_{1}-\beta_{1}\right) v}
$$

and from (6.3) and (6.4) that

$$
u^{n}=c_{2}-v
$$

It also follows that

$$
\frac{c_{1} u-\bar{\alpha}_{2}\left[1+\left(\alpha_{1}-\beta_{1}\right) v\right]}{1+\left(\alpha_{1}-\beta_{1}\right) v}=\bar{f}-\bar{\alpha}_{2} .
$$

Since zeros of $\bar{f}-\bar{\alpha}_{2}$ are $n$-fold ramified, we see that zeros of $F=c_{1} u-\bar{\alpha}_{2}\left[\left(\alpha_{1}-\right.\right.$ $\left.\beta_{1}\right) v+1$ ] are also $n$-fold ramified. Let $u_{0}$ and $v_{0}$ be two functions such that

$$
u_{0}^{n}=c_{2}-v_{0}, \quad c_{1} u_{0}-\bar{\alpha}_{2}\left[\left(\alpha_{1}-\beta_{1}\right) v_{0}+1\right]=0
$$

Eliminating $u_{0}$ from the above two equations gives

$$
\bar{\alpha}_{2}^{n}\left[\left(\alpha_{1}-\beta_{1}\right) v_{0}+1\right]^{n}+c_{1}^{n} v_{0}-c_{1}^{n} c_{2}=0
$$

i.e.,

$$
\bar{\alpha}_{2}^{n}\left[\left(\alpha_{1}-\beta_{1}\right)^{n} v_{0}^{n}+n\left(\alpha_{1}-\beta_{1}\right)^{n-1} v_{0}^{n-1}+\ldots+1\right]+c_{1}^{n} v_{0}-c_{1}^{n} c_{2}=0,
$$

from which we see that $v_{0}$ is an algebroid function of degree at most $n$. Moreover, it is seen that $c_{2}$ is not a root of (6.7). If (6.7) has only one root, then, since $n \geq 3$, by Vieta's formulas, we see that $v_{0}=-1 /\left(\alpha_{1}-\beta_{1}\right)$ and it follows from (6.7) that $-1 /\left(\alpha_{1}-\beta_{1}\right)=c_{2}$, which gives $\alpha_{2}=\beta_{1}$, a contradiction. Therefore, (6.7) has at least two distinct roots, say, $v_{1}$ and $v_{2}$. Rewrite (6.6) as

$$
-\frac{\bar{\alpha}_{2}^{n}\left[1+\left(\alpha_{1}-\beta_{1}\right) v\right]^{n}+c_{1}^{n} v-c_{1}^{n} c_{2}}{\left[1+\left(\alpha_{1}-\beta_{1}\right) v\right]^{n}}=\bar{f}^{n}-\bar{\alpha}_{2}^{n} .
$$

By a similar analysis as to (5.8) in Sect. 5, we obtain from (6.8) that $v$ is completely ramified at $v_{1}$-points and $v_{2}$-points. Moreover, from (6.4), we see that $v$ is $n$-fold ramified at poles and $c_{2}$-points, which is impossible by (2.4) since $n \geq 3$. Hence $n=2$ and we therefore have

$$
\bar{f}^{2}=\frac{c\left(f-\alpha_{1}\right)\left(f-\alpha_{2}\right)}{\left(f-\beta_{1}\right)^{2}} .
$$


In what follows, we will still use the expressions in (6.4) to transform (6.9) into (6.5) for the case $n=2$. Moreover, we have

$$
\bar{\alpha}_{2}^{2}\left[\left(\alpha_{1}-\beta_{1}\right)^{2} v_{0}^{2}+2\left(\alpha_{1}-\beta_{1}\right) v_{0}+1\right]+c_{1}^{2} v_{0}-c_{1}^{2} c_{2}=0
$$

which has two distinct roots if

$$
\left[2 \bar{\alpha}_{2}^{2}\left(\alpha_{1}-\beta_{1}\right)+c_{1}^{2}\right]^{2}-4 \bar{\alpha}_{2}^{2}\left(\alpha_{1}-\beta_{1}\right)^{2}\left(\bar{\alpha}_{2}^{2}-c_{1}^{2} c_{2}\right) \not \equiv 0
$$

Recalling that $c_{1}=\left[c\left(\alpha_{2}-\alpha_{1}\right)\right]^{1 / 2}$ and $c_{2}=1 /\left(\alpha_{2}-\alpha_{1}\right)$, we have

$$
\left[4 \bar{\alpha}_{2}^{2}\left(\alpha_{1}-\beta_{1}\right)\left(\alpha_{2}-\beta_{1}\right)+c\left(\alpha_{2}-\alpha_{1}\right)^{2}\right] c_{1}^{2} c_{2} \not \equiv 0
$$

If (6.10) holds, then $v$ must be completely ramified at poles, $c_{2}$-points, $v_{1}$-points, and $v_{2}$-points; if not, then we have

$$
4 \bar{\alpha}_{2}^{2}\left(\alpha_{1}-\beta_{1}\right)\left(\alpha_{2}-\beta_{1}\right)+c\left(\alpha_{2}-\alpha_{1}\right)^{2}=0 .
$$

Rewrite (6.9) as

$$
\bar{f}^{2}\left(f-\beta_{1}\right)^{2}=c\left(g^{2}-c_{4}^{2}\right),
$$

where $g=f-c_{3}, c_{3}=\left(\alpha_{1}+\alpha_{2}\right) / 2$ are both meromorphic functions and $c_{4}=$ $\left(\alpha_{2}-\alpha_{1}\right) / 2=1 /\left(2 c_{2}\right)$ is an algebroid function of degree at most 2. Moreover, $c_{4}^{2}=\left[\left(\alpha_{2}+\alpha_{1}\right)^{2}-4 \alpha_{1} \alpha_{2}\right] / 4$ is a meromorphic function. Put

$$
g=\frac{c_{4}}{2}\left(\beta+\frac{1}{\beta}\right)
$$

Then substitution yields

$$
\bar{f}^{2}\left(f-\beta_{1}\right)^{2}=\frac{c c_{4}^{2}}{4}\left(\beta-\frac{1}{\beta}\right)^{2},
$$

and, without loss of generality, we may suppose that

$$
\frac{c^{1 / 2} c_{4}}{2}\left(\beta-\frac{1}{\beta}\right)=\left(\bar{\alpha}_{1}+\frac{1}{\bar{v}}\right)\left[\frac{c_{4}}{2}\left(\beta+\frac{1}{\beta}\right)+c_{3}-\beta_{1}\right],
$$

i.e.,

$$
\begin{aligned}
& {\left[1-\frac{1}{c^{1 / 2}}\left(\bar{\alpha}_{1}+\frac{1}{\bar{v}}\right)\right] \beta^{2}-\frac{2\left(c_{3}-\beta_{1}\right)}{c^{1 / 2} c_{4}}\left(\bar{\alpha}_{1}+\frac{1}{\bar{v}}\right) \beta} \\
& -\left[1+\frac{1}{c^{1 / 2}}\left(\bar{\alpha}_{1}+\frac{1}{\bar{v}}\right)\right]=0 .
\end{aligned}
$$


Recall that $f=\alpha_{1}+1 / v$ and $f=g+c_{3}$. From (6.12), we have $\bar{f}^{2}=c\left(g^{2}-\right.$ $\left.c_{4}^{2}\right) /\left(f-\beta_{1}\right)^{2}$. Then the discriminant of the quadratic Eq. (6.13) with respect to $\beta$,

$$
\begin{aligned}
& \frac{4\left[\left(c_{3}-\beta_{1}\right)^{2}-c_{4}^{2}\right]}{c c_{4}^{2}} \cdot\left(\bar{\alpha}_{1}+\frac{1}{\bar{v}}\right)^{2}+4=\frac{4\left[\left(c_{3}-\beta_{1}\right)^{2}-c_{4}^{2}\right]\left(g^{2}-c_{4}^{2}\right)}{c_{4}^{2}\left(g+c_{3}-\beta_{1}\right)^{2}}+4 \\
& =\frac{4\left[\left(c_{3}-\beta_{1}\right) g+c_{4}^{2}\right]^{2}}{c_{4}^{2}\left(g+c_{3}-\beta_{1}\right)^{2}}
\end{aligned}
$$

implies that $\beta$ is meromorphic apart from at most $S(r, f)$ many points. Moreover, roots of the equation

$$
\begin{aligned}
& {\left[4\left(\alpha_{1}-\beta_{1}\right)\left(\alpha_{2}-\beta_{1}\right) \bar{\alpha}_{1}^{2}+c\left(\alpha_{2}-\alpha_{1}\right)^{2}\right] \bar{v}^{2}} \\
& \quad+8\left(\alpha_{1}-\beta_{1}\right)\left(\alpha_{2}-\beta_{1}\right) \bar{\alpha}_{1} \bar{v}+4\left(\alpha_{1}-\beta_{1}\right)\left(\alpha_{2}-\beta_{1}\right)=0
\end{aligned}
$$

with respect to $\bar{v}$ must be $c_{2}$ or coincide with $v_{1}, v_{2}$.

If $c_{2}=1 /\left(\alpha_{2}-\alpha_{1}\right)$ is a root of (6.14), then we have

$$
4\left(\alpha_{1}-\beta_{1}\right)\left(\alpha_{2}-\beta_{1}\right)\left[\bar{\alpha}_{1}^{2}+2 \bar{\alpha}_{1}\left(\bar{\alpha}_{2}-\bar{\alpha}_{1}\right)+\left(\bar{\alpha}_{2}-\bar{\alpha}_{1}\right)^{2}\right]+c\left(\alpha_{2}-\alpha_{1}\right)^{2}=0,
$$

which yields Eq. (6.11). On the other hand, by exchanging the roles of $\alpha_{1}$ and $\alpha_{2}$, we also have

$$
4 \bar{\alpha}_{1}^{2}\left(\alpha_{1}-\beta_{1}\right)\left(\alpha_{2}-\beta_{1}\right)+c\left(\alpha_{2}-\alpha_{1}\right)^{2}=0 .
$$

From (6.11) and (6.15), we see that $\alpha_{2}=-\alpha_{1}$, and it follows that $c=\bar{\alpha}_{1}^{2}\left(1-\beta_{1}^{2} / \alpha_{1}^{2}\right)$. Set $\delta=\beta_{1} / \alpha_{1}$. Then $\delta \neq \pm 1$ is an algebroid function of degree at most 2. By doing the transformation $f \rightarrow \alpha_{1} f$, (6.9) becomes

$$
\bar{f}^{2}=\frac{\left(1-\delta^{2}\right)\left(f^{2}-1\right)}{(f-\delta)^{2}}=1-\left(\frac{\delta f-1}{f-\delta}\right)^{2}
$$

which is the Eq. (3.7).

If $c_{2}=1 /\left(\alpha_{2}-\alpha_{1}\right)$ is not a root of (6.14), then roots of (6.14) must coincide with $v_{1}, v_{2}$, which means that

$$
\begin{aligned}
& \frac{\overline{\bar{\alpha}}_{2}^{2}\left(\bar{\alpha}_{1}-\bar{\beta}_{1}\right)^{2}}{4\left(\alpha_{1}-\beta_{1}\right)\left(\alpha_{2}-\beta_{1}\right) \bar{\alpha}_{1}^{2}+c\left(\alpha_{2}-\alpha_{1}\right)^{2}}=\frac{2 \overline{\bar{\alpha}}_{2}^{2}\left(\bar{\alpha}_{1}-\bar{\beta}_{1}\right)+\bar{c}_{1}^{2}}{8\left(\alpha_{1}-\beta_{1}\right)\left(\alpha_{2}-\beta_{1}\right) \bar{\alpha}_{1}} \\
& =\frac{\overline{\bar{\alpha}}_{2}^{2}-\bar{c}_{1}^{2} \bar{c}_{2}}{4\left(\alpha_{1}-\beta_{1}\right)\left(\alpha_{2}-\beta_{1}\right)} .
\end{aligned}
$$

Recall that $c_{1}=\left[c\left(\alpha_{2}-\alpha_{1}\right)\right]^{1 / 2}$ and $c_{2}=1 /\left(\alpha_{2}-\alpha_{1}\right)$. We obtain from the second equation of (6.16) that

$$
\left(\bar{\alpha}_{1}+\bar{\alpha}_{2}\right) \bar{c}=2 \overline{\bar{\alpha}}_{2}^{2} \bar{\beta}_{1}
$$


By exchanging the roles of $\alpha_{1}$ and $\alpha_{2}$, we see that $\bar{\beta}_{1} \equiv 0$, and then it follows from the first equation of (6.16) that

$$
\bar{c} c=\bar{c}_{1}^{2}+c \overline{\bar{\alpha}}_{1}^{2}
$$

Set $d=c / \bar{\alpha}_{1}^{2}$. Then $d$ is a meromorphic function satisfying $\bar{d} d=\bar{d}+d$. By doing the transformation $f \rightarrow \alpha_{1} f$, (6.9) becomes

$$
\bar{f}^{2}=d\left(1-f^{-2}\right)
$$

which is the Eq. (3.8).

We now look at Eq. (6.2). By using a similar analysis on the $\alpha_{i}$-points of $f$ as on the $\alpha_{i}$-points of $f$ in (5.2) in Sect. 5, we obtain the following three possibilities:

$$
\begin{aligned}
& \bar{f}^{3}=\frac{c\left(f-\alpha_{1}\right)\left(f-\alpha_{2}\right)\left(f-\alpha_{3}\right)}{\left(f-\beta_{1}\right)^{3}}, \quad \alpha_{1} \alpha_{2} \alpha_{3} \not \equiv 0, \\
& \bar{f}^{4}=\frac{c\left(f-\alpha_{1}\right)^{2}\left(f-\alpha_{2}\right)\left(f-\alpha_{3}\right)}{\left(f-\beta_{1}\right)^{4}}, \quad \alpha_{1} \alpha_{2} \alpha_{3} \not \equiv 0, \\
& \bar{f}^{6}=\frac{c\left(f-\alpha_{1}\right)^{3}\left(f-\alpha_{2}\right)^{2}\left(f-\alpha_{3}\right)}{\left(f-\beta_{1}\right)^{6}}, \quad \alpha_{1} \alpha_{2} \alpha_{3} \not \equiv 0 .
\end{aligned}
$$

Note that $\alpha_{1}$ in (6.18) and $\alpha_{1}, \alpha_{2}, \alpha_{3}$ in (6.19) and $\beta_{1}$ in these three equations are all meromorphic functions.

Let's first consider Eq. (6.17). Put

$$
\frac{\bar{f}\left(f-\beta_{1}\right)}{f-\alpha_{1}}=c_{1} u, \quad \frac{1}{f-\alpha_{1}}=b_{2} v+b_{1},
$$

where $u$ and $v$ are two algebroid functions with at most $S(r, f)$ many branch points and the coefficients $c_{1}, b_{1}, b_{2}$ take the same form as in (5.20) which are algebroid functions of degrees at most 9, 3, 3, respectively. Note that all the coefficients of (6.17) are small functions of $v$. Then we have

$$
f=\alpha_{1}+\frac{1}{b_{2} v+b_{1}}, \quad \bar{f}=\frac{c_{1} u}{1+\left(\alpha_{1}-\beta_{1}\right)\left(b_{2} v+b_{1}\right)}
$$

and from (6.17) and (6.20) that

$$
v^{2}=4 u^{3}+1
$$

It also follows that

$$
\frac{c_{1} u-\bar{\alpha}_{2}\left[1+\left(\alpha_{1}-\beta_{1}\right)\left(b_{2} v+b_{1}\right)\right]}{1+\left(\alpha_{1}-\beta_{1}\right)\left(b_{2} v+b_{1}\right)}=\bar{f}-\bar{\alpha}_{2} .
$$

Since zeros of $\bar{f}-\bar{\alpha}_{2}$ are threefold ramified, we see that zeros of $F=c_{1} u-\bar{\alpha}_{2}[1+$ $\left.\left(\alpha_{1}-\beta_{1}\right)\left(b_{2} v+b_{1}\right)\right]$ are also threefold ramified. Let $u_{0}$ and $v_{0}$ be two functions such 
that

$$
v_{0}^{2}=4 u_{0}^{3}+1, \quad c_{1} u_{0}-\bar{\alpha}_{2}\left[1+\left(\alpha_{1}-\beta_{1}\right)\left(b_{2} v_{0}+b_{1}\right)\right]=0 .
$$

Eliminating $u_{0}$ from the above two equations gives

$$
4 \bar{\alpha}_{2}^{3}\left[1+\left(\alpha_{1}-\beta_{1}\right)\left(b_{2} v_{0}+b_{1}\right)\right]^{3}-c_{1}^{3}\left(v_{0}^{2}-1\right)=0,
$$

from which we see that $v_{0}$ is an algebroid function of degree at most 27. Moreover, it is seen that \pm 1 are not roots of (6.23), for otherwise we have $1+\left(\alpha_{1}-\beta_{1}\right)\left(b_{2} v_{0}+b_{1}\right)=0$ and it follows that $\pm b_{2}+b_{1}=-1 /\left(\alpha_{1}-\beta_{1}\right)$, which gives $\alpha_{2}=\beta_{1}$ or $\alpha_{3}=\beta_{1}$, a contradiction. Rewrite (6.22) as

$$
-\frac{4 \bar{\alpha}_{2}^{3}\left[1+\left(\alpha_{1}-\beta_{1}\right)\left(b_{2} v+b_{1}\right)\right]^{3}-c_{1}^{3}\left(v^{2}-1\right)}{4\left[1+\left(\alpha_{1}-\beta_{1}\right)\left(b_{2} v+b_{1}\right)\right]^{3}}=\bar{f}^{3}-\bar{\alpha}_{2}^{3} .
$$

From Eq. (6.21), we see that $v$ is threefold ramified over $\pm 1, \infty$. This implies that (6.23) can admit only one root, for otherwise by a similar analysis as to (5.8) in Sect. 5 we obtain from (6.24) that (6.23) has at least two roots, say, $v_{1}$ and $v_{2}$, distinct from $\pm 1, \infty$ such that $v$ is completely ramified at $v_{1}$-points and $v_{2}$-points, which is impossible by Theorem 1. Expanding (6.23) gives

$$
\begin{aligned}
& 4 \bar{\alpha}_{2}^{3}\left(\alpha_{1}-\beta_{1}\right)^{3} b_{2}^{3} v_{0}^{3}+12 \bar{\alpha}_{2}^{3}\left(\alpha_{1}-\beta_{1}\right)^{2} b_{2}^{2}\left[b_{1}\left(\alpha_{1}-\beta_{1}\right)+1\right] v_{0}^{2}-c_{1}^{3} v_{0}^{2} \\
& \quad+12 \bar{\alpha}_{2}^{3}\left(\alpha_{1}-\beta_{1}\right) b_{2}\left[b_{1}\left(\alpha_{1}-\beta_{1}\right)+1\right]^{2} v_{0}+4 \bar{\alpha}_{2}^{3}\left[b_{1}\left(\alpha_{1}-\beta_{1}\right)+1\right]^{3}+c_{1}^{3}=0,
\end{aligned}
$$

and so by Vieta's formulas, we get

$$
\frac{12 \bar{\alpha}_{2}^{3}\left(\alpha_{1}-\beta_{1}\right)^{2} b_{2}^{2}\left[b_{1}\left(\alpha_{1}-\beta_{1}\right)+1\right]-c_{1}^{3}}{4 \bar{\alpha}_{2}^{3}\left(\alpha_{1}-\beta_{1}\right)^{3} b_{2}^{3}}=-3 v_{0}
$$

and

$$
\frac{3\left[b_{1}\left(\alpha_{1}-\beta_{1}\right)+1\right]^{2}}{\left(\alpha_{1}-\beta_{1}\right)^{2} b_{2}^{2}}=3 v_{0}^{2} .
$$

From (6.26), we have $v_{0}= \pm\left[b_{1}\left(\alpha_{1}-\beta_{1}\right)+1\right] /\left[\left(\alpha_{1}-\beta_{1}\right) b_{2}\right]$. If $v_{0}=-\left[b_{1}\left(\alpha_{1}-\right.\right.$ $\left.\left.\beta_{1}\right)+1\right] /\left[\left(\alpha_{1}-\beta_{1}\right) b_{2}\right]$, then substitution into (6.23) yields $v_{0}^{2}=1$, a contradiction. Hence $v_{0}=\left[b_{1}\left(\alpha_{1}-\beta_{1}\right)+1\right] /\left[\left(\alpha_{1}-\beta_{1}\right) b_{2}\right]$. Combining this equation with (6.25), we get

$$
c_{1}^{3}=24 \bar{\alpha}_{2}^{3}\left(\alpha_{1}-\beta_{1}\right)^{2} b_{2}^{2}\left[b_{1}\left(\alpha_{1}-\beta_{1}\right)+1\right] .
$$

Similarly, the above equation also holds when replacing $\bar{\alpha}_{2}$ in (6.22) with $\bar{\alpha}_{1}$ and $\bar{\alpha}_{3}$, which implies that $\alpha_{1}^{3}=\alpha_{2}^{3}=\alpha_{3}^{3}$. Thus, $\alpha_{2}=\eta \alpha_{1}, \alpha_{3}=\eta^{2} \alpha_{1}$, where $\eta$ is the cubic root of 1 satisfying $\eta^{2}+\eta+1=0$. Moreover, we have

$$
c_{1}^{3}=4 c b_{2}^{2}\left(\alpha_{2}-\alpha_{1}\right)\left(\alpha_{3}-\alpha_{1}\right)=24 \bar{\alpha}_{1}^{3}\left(\alpha_{1}-\beta_{1}\right)^{2} b_{2}^{2}\left[b_{1}\left(\alpha_{1}-\beta_{1}\right)+1\right] .
$$

Set $\gamma_{1}=\beta_{1} / \alpha_{1}, \gamma_{1}^{3} \not \equiv 1$. Using equation $\eta^{2}+\eta+1=0$, we get from the above equation that

$$
c=\bar{\alpha}_{1}^{3}\left(1-\gamma_{1}\right)^{2}\left(1+\gamma_{1}\right) .
$$


Setting $\gamma_{2}=\beta_{1} / \alpha_{2}=\beta_{1} /\left(\eta \alpha_{1}\right), \gamma_{3}=\beta_{1} / \alpha_{3}=\beta_{1} /\left(\eta^{2} \alpha_{1}\right)$, we have $\gamma_{2} \not \equiv 1, \gamma_{3} \not \equiv 1$. Exchanging the role of $\alpha_{1}$ to $\alpha_{2}$ and $\alpha_{3}$, respectively, we also have

$$
c=\bar{\alpha}_{3}^{3}\left(1-\eta \gamma_{1}\right)^{2}\left(1+\eta \gamma_{1}\right)=\bar{\alpha}_{2}^{3}\left(1-\eta^{2} \gamma_{1}\right)^{2}\left(1+\eta^{2} \gamma_{1}\right),
$$

which together with the equation $\eta^{2}+\eta+1=0$ yields

$$
\left(\eta^{2}-\eta\right) \gamma_{1}\left(\gamma_{1}-1\right)=0
$$

Therefore, we have $\gamma_{1} \equiv 0$ and so $\beta_{1} \equiv 0$ and then from (6.27) that $c=\bar{\alpha}_{1}^{3}$. By doing the transformation $f \rightarrow \alpha_{1} f$, (6.17) becomes

$$
\bar{f}^{3}=1-f^{-3}
$$

which is the Eq. (3.14).

We now consider Eq. (6.18). Put

$$
\frac{\bar{f}\left(f-\beta_{1}\right)}{f-\alpha_{1}}=c_{1} u, \quad \frac{1}{f-\alpha_{1}}=b_{2} v+b_{1},
$$

where $u$ and $v$ are two algebroid functions with at most $S(r, f)$ many branch points and the coefficients $c_{1}, b_{1}, b_{2}$ take the same form as in (5.27) which are algebroid functions of degrees at most 8, 2, 2, respectively. Note that all the coefficients of (6.18) are small functions of $v$. Then we have

$$
f=\frac{1}{b_{2} v+b_{1}}+\alpha_{1}, \quad \bar{f}=\frac{c_{1} u}{1+\left(\alpha_{1}-\beta_{1}\right)\left(b_{2} v+b_{1}\right)}
$$

and from (6.18) and (6.28) that

$$
v^{2}=1-u^{4}
$$

It also follows that

$$
\frac{c_{1} u-\bar{\alpha}_{2}\left[1+\left(\alpha_{1}-\beta_{1}\right)\left(b_{2} v+b_{1}\right)\right]}{1+\left(\alpha_{1}-\beta_{1}\right)\left(b_{2} v+b_{1}\right)}=\bar{f}-\bar{\alpha}_{2} .
$$

Since zeros of $\bar{f}-\bar{\alpha}_{2}$ are fourfold ramified, we see that zeros of $F=c_{1} u-\bar{\alpha}_{2}[1+$ $\left.\left(\alpha_{1}-\beta_{1}\right)\left(b_{2} v+b_{1}\right)\right]$ are also fourfold ramified. Let $u_{0}$ and $v_{0}$ be functions such that

$$
v_{0}^{2}=1-u_{0}^{4}, \quad c_{1} u_{0}-\bar{\alpha}_{2}\left[1+\left(\alpha_{1}-\beta_{1}\right)\left(b_{2} v_{0}+b_{1}\right)\right]=0 .
$$

Eliminating $u_{0}$ from the above two equations gives

$$
\bar{\alpha}_{2}^{4}\left[1+\left(\alpha_{1}-\beta_{1}\right)\left(b_{2} v_{0}+b_{1}\right)\right]^{4}-c_{1}^{4}\left(1-v_{0}^{2}\right)=0,
$$

from which we see that $v_{0}$ is an algebroid function of degree at most 16 . Moreover, it is seen that \pm 1 are not roots of (6.31), for otherwise we have $1+\left(\alpha_{1}-\beta_{1}\right)\left(b_{2} v_{0}+b_{1}\right)=0$ 
and it follows that $\pm b_{2}+b_{1}=-1 /\left(\alpha_{1}-\beta_{1}\right)$, which gives $\alpha_{2}=\beta_{1}$ or $\alpha_{3}=\beta_{1}$, a contradiction. Rewrite (6.30) as

$$
\frac{c_{1}^{4}\left(1-v^{2}\right)-\bar{\alpha}_{2}^{4}\left[1+\left(\alpha_{1}-\beta_{1}\right)\left(b_{2} v+b_{1}\right)\right]^{4}}{\left[1+\left(\alpha_{1}-\beta_{1}\right)\left(b_{2} v+b_{1}\right)\right]^{4}}=\bar{f}^{4}-\bar{\alpha}_{2}^{4} .
$$

From Eq. (6.29), we see that $v$ is completely ramified over $\pm 1, \infty$. This implies that (6.31) can admit only one root, for otherwise by a similar analysis as to (5.8) in Sect. 5 we obtain from (6.32) that (6.31) has at least two roots, say, $v_{1}$ and $v_{2}$, distinct from $\pm 1, \infty$ such that $v$ is completely ramified at $v_{1}$-points and $v_{2}$-points, which is impossible by Theorem 1. Expanding (6.31) gives

$$
\begin{gathered}
\bar{\alpha}_{2}^{4}\left\{\left(\alpha_{1}-\beta_{1}\right)^{4} b_{2}^{4} v_{0}^{4}+4\left(\alpha_{1}-\beta_{1}\right)^{3} b_{2}^{3}\left[1+\left(\alpha_{1}-\beta_{1}\right) b_{1}\right] v_{0}^{3}\right. \\
\left.+\cdots+\left[1+\left(\alpha_{1}-\beta_{1}\right) b_{1}\right]^{4}\right\}-c_{1}^{4}\left(1-v_{0}^{2}\right)=0,
\end{gathered}
$$

and so by Vieta's formulas, we obtain $4\left[1+\left(\alpha_{1}-\beta_{1}\right) b_{1}\right] /\left(\alpha_{1}-\beta_{1}\right) b_{2}=-4 v_{0}$, which gives $1+\left(\alpha_{1}-\beta_{1}\right)\left(b_{2} v_{0}+b_{1}\right)=0$ and it follows from (6.31) that $v_{0}^{2}=1$, a contradiction. Therefore, (6.18) cannot admit any meromorphic solutions.

Finally, let's consider Eq. (6.19). Put

$$
\frac{\bar{f}\left(f-\beta_{1}\right)}{f-\alpha_{1}}=c_{1} u, \quad \frac{1}{f-\alpha_{1}}=b_{2} v-b_{1}
$$

where $u$ is an algebroid function with at most $S(r, f)$ many branch points and the coefficients $c_{1}, b_{1}, b_{2}$ take the same form as in (5.33) and $c_{1}$ is an algebroid function of degree at most 6. Note that all the coefficients of (6.19) are small functions of $v$. Then we have

$$
f=\frac{1}{b_{2} v-b_{1}}+\alpha_{1}, \quad \bar{f}=\frac{c_{1} u}{1+\left(\alpha_{1}-\beta_{1}\right)\left(b_{2} v-b_{1}\right)}
$$

and from (6.19) and (6.33) that

$$
u^{6}=(v-1)^{2}(v+2)=v^{3}-3 v+2 .
$$

It also follows that

$$
\frac{c_{1} u-\bar{\alpha}_{3}\left[1+\left(\alpha_{1}-\beta_{1}\right)\left(b_{2} v-b_{1}\right)\right]}{1+\left(\alpha_{1}-\beta_{1}\right)\left(b_{2} v-b_{1}\right)}=\bar{f}-\bar{\alpha}_{3} .
$$

Since zeros of $\bar{f}-\bar{\alpha}_{3}$ are sixfold ramified, we see that zeros of $F=c_{1} u-\bar{\alpha}_{3}[1+$ $\left.\left(\alpha_{1}-\beta_{1}\right)\left(b_{2} v-b_{1}\right)\right]$ are also sixfold ramified. Let $u_{0}$ and $v_{0}$ be functions such that

$$
u_{0}^{6}=v_{0}^{3}-3 v_{0}+2, \quad c_{1} u_{0}-\bar{\alpha}_{3}\left[1+\left(\alpha_{1}-\beta_{1}\right)\left(b_{2} v_{0}-b_{1}\right)\right]=0 .
$$


Eliminating $u_{0}$ from the above two equations gives

$$
\bar{\alpha}_{3}^{6}\left[1+\left(\alpha_{1}-\beta_{1}\right)\left(b_{2} v_{0}-b_{1}\right)\right]^{6}-c_{1}^{6}\left(v_{0}^{3}-3 v_{0}+2\right)=0,
$$

from which we see that $v_{0}$ is an algebroid function of degree at most 6. Moreover, it is easy to see that 1 and -2 are not roots of (6.36), for otherwise we have $1+\left(\alpha_{1}-\beta_{1}\right)\left(b_{2}-\right.$ $\left.b_{1}\right)=0$, which gives $\alpha_{2}=\beta_{1}$, a contradiction; or $1+\left(\alpha_{1}-\beta_{1}\right)\left(-2 b_{2}-b_{1}\right)=0$, which gives $\alpha_{3}=\beta_{1}$, a contradiction. Rewrite (6.35) as

$$
\frac{c_{1}^{6}\left(v^{3}-3 v+2\right)-\bar{\alpha}_{3}^{6}\left[1+\left(\alpha_{1}-\beta_{1}\right)\left(b_{2} v-b_{1}\right)\right]^{6}}{\left[1+\left(\alpha_{1}-\beta_{1}\right)\left(b_{2} v-b_{1}\right)\right]^{6}}=\bar{f}^{6}-\bar{\alpha}_{3}^{6} .
$$

From Eq. (6.34), we see that $v$ is completely ramified over $1,-2, \infty$. This implies that (6.36) can admit only one root, for otherwise by a similar analysis as to (5.8) in Sect. 5 we obtain from (6.37) that (6.36) has at least two roots, say, $v_{1}$ and $v_{2}$, distinct from $1,-2, \infty$ such that $v$ is completely ramified at $v_{1}$-points and $v_{2}$-points, which is impossible by Theorem 1. Expanding (6.36) gives

$$
\begin{gathered}
\bar{\alpha}_{3}^{6}\left\{\left(\alpha_{1}-\beta_{1}\right)^{6} b_{2}^{6} v_{0}^{6}+6\left(\alpha_{1}-\beta_{1}\right)^{5} b_{2}^{5} v_{0}^{5}\left[1-\left(\alpha_{1}-\beta_{1}\right) b_{1}\right]\right. \\
\left.+\cdots+\left[1-\left(\alpha_{1}-\beta_{1}\right) b_{1}\right]^{6}\right\}-c_{1}^{6}\left(v_{0}^{3}-3 v_{0}+2\right)=0,
\end{gathered}
$$

and so by Vieta's formulas, we obtain $-6\left[1-\left(\alpha_{1}-\beta_{1}\right) b_{1}\right] /\left(\alpha_{1}-\beta_{1}\right) b_{2}=-6 v_{0}$, which gives $1+\left(\alpha_{1}-\beta_{1}\right)\left(b_{2} v_{0}-b_{1}\right)=0$ and it follows from (6.36) that $v_{0}^{3}-3 v_{0}+2=0$, a contradiction. Therefore, (6.19) cannot admit any meromorphic solutions.

\subsection{Q(z, f) has Two Distinct Roots}

Now $P(z, f)$ can have one or two roots. We discuss these two cases separately as follows:

Case 1: $P(z, f)$ has only one root. Then (3.2) assumes the following form:

$$
\bar{f}^{n}=\frac{c\left(f-\alpha_{1}\right)^{n}}{\left(f-\beta_{1}\right)^{\nu_{1}}\left(f-\beta_{2}\right)^{\nu_{2}}},
$$

where $v_{1}, v_{2}$ are positive integers satisfying $v_{1}+v_{2}=n$. By doing a bilinear transformation $f \rightarrow 1 / f$, if $\alpha_{1} \equiv 0$, we obtain (5.1); if $\alpha_{1} \neq \equiv 0$ and $\beta_{1}, \beta_{2} \not \equiv 0$, we obtain (6.1). So in what follows we only consider the case when $\alpha_{1} \not \equiv 0$ and $\beta_{1} \equiv 0$. Note that $\left(v_{1}, v_{2}\right)=1$. If $n=2$, then obviously $v_{1}=v_{2}=1$; if $n \geq 3$, then by using a similar analysis on the $\beta_{j}$-points of $f$ in (6.38) as to $\alpha_{i}$-points of $f$ in (5.1) in Sect. 5, we obtain that $v_{1}=1$ or $\nu_{2}=1$. Thus (6.38) is one of the following forms:

$$
\begin{aligned}
& \bar{f}^{n}=\frac{c\left(f-\alpha_{1}\right)^{n}}{f\left(f-\beta_{2}\right)^{n-1}}, \\
& \bar{f}^{n}=\frac{c\left(f-\alpha_{1}\right)^{n}}{f^{n-1}\left(f-\beta_{2}\right)} .
\end{aligned}
$$


Consider Eq. (6.39) first. By doing a bilinear transformation $f \rightarrow 1 / f$, we obtain

$$
\bar{f}^{n}=\frac{d_{1}\left(f-\gamma_{1}\right)^{n-1}}{\left(f-\lambda_{1}\right)^{n}},
$$

where $d_{1}=-\beta_{2}^{n-1} /\left(c \alpha_{1}^{n}\right), \gamma_{1}=1 / \beta_{2}$, and $\lambda_{1}=1 / \alpha_{1}$ are nonzero small meromorphic functions of $f$. Moreover, it is seen that $f$ is completely ramified at poles, $\gamma_{1}$-points and also $\lambda_{1}$-points. Set $c_{1}=d_{1}^{1 / n}$. Then $c_{1}$ is an algebroid function of degree at most $n$. Put

$$
c_{1} u=\frac{\bar{f}\left(f-\lambda_{1}\right)}{f-\gamma_{1}}, \quad v=\frac{1}{f-\gamma_{1}},
$$

where $u$ is an algebroid function with at most $S(r, f)$ many branch points. By the Valiron-Mohon'ko identity [15,26] (see also [13]), we have $T(r, v)=T(r, f)+$ $S(r, f)$ and thus all the coefficients of (6.39) are small functions of $v$. Then we have

$$
f=\frac{1}{v}+\gamma_{1}, \quad \bar{f}=\frac{c_{1} u}{1+\left(\gamma_{1}-\lambda_{1}\right) v}
$$

and from (6.41) and (6.42) that

$$
u^{n}=v
$$

It also follows that

$$
\frac{c_{1} u-\bar{\gamma}_{1}\left[1+\left(\gamma_{1}-\lambda_{1}\right) v\right]}{1+\left(\gamma_{1}-\lambda_{1}\right) v}=\bar{f}-\bar{\gamma}_{1} .
$$

Since $\bar{f}$ is completely ramified at $\bar{\gamma}_{1}$-points, we see that $F=c_{1} u-\bar{\gamma}_{1}\left[\left(\gamma_{1}-\lambda_{1}\right) v+1\right]$ is completely ramified at zeros. Let $u_{0}$ and $v_{0}$ be functions such that

$$
u_{0}^{n}=v_{0}, \quad c_{1} u_{0}-\bar{\gamma}_{1}\left[\left(\gamma_{1}-\lambda_{1}\right) v_{0}+1\right]=0
$$

Eliminating $u_{0}$ from the above two equations gives

$$
\bar{\gamma}_{1}^{n}\left[\left(\gamma_{1}-\lambda_{1}\right) v_{0}+1\right]^{n}-c_{1}^{n} v_{0}=0
$$

i.e.,

$$
\bar{\gamma}_{1}^{n}\left[\left(\gamma_{1}-\lambda_{1}\right)^{n} v_{0}^{n}+n\left(\gamma_{1}-\lambda_{1}\right)^{n-1} v_{0}^{n-1}+\ldots+1\right]-c_{1}^{n} v_{0}=0,
$$

from which we see that $v_{0}$ is an algebroid function of degree at most $n$. Moreover, it is seen that 0 and $1 /\left(\lambda_{1}-\gamma_{1}\right)$ are not roots of (6.44). Rewrite (6.43) as

$$
\frac{c_{1}^{n} v-\bar{\gamma}_{1}^{n}\left[1+\left(\gamma_{1}-\lambda_{1}\right) v\right]^{n}}{\left[1+\left(\gamma_{1}-\lambda_{1}\right) v\right]^{n}}=\bar{f}^{n}-\bar{\gamma}_{1}^{n} .
$$

We see from (6.42) that $v$ is completely ramified at zeros, poles, and $1 /\left(\lambda_{1}-\gamma_{1}\right)$ points. This implies that (6.44) can admit only one root, for otherwise by using a similar analysis as to (5.8) in Sect. 5 we obtain from (6.45) that (6.44) has at least two 
roots, say, $v_{1}$ and $v_{2}$, distinct from $0, \infty, 1 /\left(\lambda_{1}-\gamma_{1}\right)$ such that $v$ is completely ramified at $v_{1}$-points and $v_{2}$-points, which is impossible by Theorem 1 . However, when $n \geq 3$, by Vieta's formulas we see that the only root is $v_{0}=-1 /\left(\gamma_{1}-\lambda_{1}\right)$ and it follows from (6.44) that $c_{1}^{n} /\left(\gamma_{1}-\lambda_{1}\right)=0$, a contradiction. Hence $n=2$ and we therefore have

$$
\bar{f}^{2}=\frac{c\left(f-\gamma_{1}\right)}{\left(f-\lambda_{1}\right)^{2}} .
$$

Moreover, we conclude from the above reasoning that the quadratic equation

$$
\bar{\gamma}_{1}^{2}\left[\left(\gamma_{1}-\lambda_{1}\right) v_{0}+1\right]^{2}-c_{1}^{2} v_{0}=0
$$

can admit only one root. It follows that the discriminant of Eq. (6.47) with respect to $v_{0}$ satisfies

$$
\left[2 \bar{\gamma}_{1}^{2}\left(\gamma_{1}-\lambda_{1}\right)-c_{1}^{2}\right]^{2}-4 \bar{\gamma}_{1}^{4}\left(\gamma_{1}-\lambda_{1}\right)^{2}=0
$$

i.e.,

$$
d_{1}=4 \bar{\gamma}_{1}^{2}\left(\gamma_{1}-\lambda_{1}\right)
$$

On the other hand, since $\bar{f}$ is completely ramified at $\bar{\lambda}_{1}$-points, if we consider equation

$$
\frac{c_{1} u-\bar{\lambda}_{1}\left[1+\left(\gamma_{1}-\lambda_{1}\right) v\right]}{1+\left(\gamma_{1}-\lambda_{1}\right) v}=\bar{f}-\bar{\lambda}_{1},
$$

then by similar arguments as above we also have

$$
d_{1}=4 \bar{\lambda}_{1}^{2}\left(\gamma_{1}-\lambda_{1}\right)
$$

From (6.48) and (6.49), we see that $\lambda_{1}=-\gamma_{1}$ and it follows that $d_{1}=-8 \bar{\lambda}_{1}^{2} \lambda_{1}$. By doing the transformation $f \rightarrow \gamma_{1} f$, then (6.46) becomes

$$
\bar{f}^{2}=-\frac{8(f+1)}{(f-1)^{2}}=1-\left(\frac{f+3}{f-1}\right)^{2},
$$

which is the Eq. (3.9).

Consider now Eq. (6.40). By doing a bilinear transformation $f \rightarrow 1 / f$, (6.40) becomes

$$
\bar{f}^{n}=\frac{d_{2}\left(f-\gamma_{2}\right)}{\left(f-\lambda_{2}\right)^{n}},
$$

where $d_{2}=(-1)^{1-n} \beta_{1} /\left(c \alpha_{1}^{n}\right), \gamma_{2}=1 / \beta_{1}$, and $\lambda_{2}=1 / \alpha_{1}$ are nonzero small meromorphic functions of $f$. Moreover, $f$ is completely ramified at poles, $\gamma_{2}$-points and also $\lambda_{2}$-points. By a similar reasoning as above, we can also obtain $n=2$ and this will lead (6.50) into (3.9) again. We omit all those details.

Case 2: $P(z, f)$ has two distinct roots. Then (3.2) assumes the following form:

$$
\bar{f}^{n}=\frac{c\left(f-\alpha_{1}\right)^{\kappa_{1}}\left(f-\alpha_{2}\right)^{\kappa_{2}}}{\left(f-\beta_{1}\right)^{\nu_{1}}\left(f-\beta_{2}\right)^{\nu_{2}}}, \quad \alpha_{1} \alpha_{2} \not \equiv 0,
$$


where $\kappa_{i}, v_{j}$ are positive integers satisfying $\kappa_{1}+\kappa_{2}=n, v_{1}+v_{2}=n$. Set $k_{1}=\left(n, \kappa_{1}\right)$, $k_{2}=\left(n, \kappa_{2}\right), l_{1}=\left(n, v_{1}\right), l_{2}=\left(n, v_{2}\right)$ and let $K_{1}=n / k_{1}, K_{2}=n / k_{2}, L_{1}=n / l_{1}$, $L_{2}=n / l_{2}$. Obviously, $K_{1} \geq 2, K_{2} \geq 2, L_{1} \geq 2, L_{2} \geq 2$. Now $f$ is $K_{i}$-fold ramified at $\alpha_{i}$-points and $L_{j}$-fold ramified at $\beta_{j}$-points, and by (2.4) we conclude that $K_{1}=K_{2}=L_{1}=L_{2}=2$ and $k_{1}+k_{2}=n, l_{1}+l_{2}=n$. Since $\kappa_{1}+\kappa_{2}=n$, $v_{1}+v_{2}=n$, we see that $\kappa_{1}=\kappa_{2}=v_{1}=v_{2}=n / 2$. Hence $n=2$ and we therefore have

$$
\bar{f}^{2}=\frac{c\left(f-\alpha_{1}\right)\left(f-\alpha_{2}\right)}{\left(f-\beta_{1}\right)\left(f-\beta_{2}\right)}, \quad \alpha_{1} \alpha_{2} \not \equiv 0 .
$$

Now $\alpha_{i}, \beta_{j}$ are algebroid functions of degree at most 2. Moreover, if $\beta_{1} \equiv 0$ or $\beta_{2} \equiv 0$, then $f$ will be fourfold ramified at $\alpha_{i}$-points, which yields a contradiction to (2.4). Therefore, $\beta_{1} \not \equiv 0$ and $\beta_{2} \not \equiv 0$.

Set $c_{1}=c^{1 / 2}$. Then $c_{1}$ is an algebroid function of degree at most 2. Put

$$
c_{1} u=\frac{\bar{f}\left(f-\beta_{1}\right)}{f-\alpha_{1}}, \quad v=\frac{f-\beta_{1}}{f-\alpha_{1}}
$$

where $u$ and $v$ are two algebroid functions with at most $S(r, f)$ many branch points. By the Valiron-Mohon'ko identity [15,26] (see also [13]), we have $T(r, v)=T(r, f)+$ $S(r, f)$ and thus all the coefficients of (6.51) are small functions of $v$. Then we have

$$
f=\frac{\alpha_{1} v-\beta_{1}}{v-1}, \quad \bar{f}=\frac{c_{1} u}{v}
$$

and from (6.51) and (6.52) that

$$
u^{2}=v \frac{\left(\alpha_{1}-\alpha_{2}\right) v-\left(\beta_{1}-\alpha_{2}\right)}{\left(\alpha_{1}-\beta_{2}\right) v-\left(\beta_{1}-\beta_{2}\right)} .
$$

It also follows that

$$
\frac{c_{1} u-\bar{\alpha}_{1} v}{v}=\bar{f}-\bar{\alpha}_{1}
$$

Since zeros of $\bar{f}-\bar{\alpha}_{1}$ are twofold ramified, we see that zeros of $F=c_{1} u-\bar{\alpha}_{1} v$ are also twofold ramified. Let $u_{0}$ and $v_{0}$ be functions such that

$$
u_{0}^{2}=v_{0} \frac{\left(\alpha_{1}-\alpha_{2}\right) v_{0}-\left(\beta_{1}-\alpha_{2}\right)}{\left(\alpha_{1}-\beta_{2}\right) v_{0}-\left(\beta_{1}-\beta_{2}\right)}, \quad c_{1} u_{0}-\bar{\alpha}_{1} v_{0}=0 .
$$

Eliminating $u_{0}$ from the above two equations gives $v_{0} \equiv 0$ or

$$
\bar{\alpha}_{1}^{2}\left(\alpha_{1}-\beta_{2}\right) v_{0}^{2}+c_{1}^{2}\left(\beta_{1}-\alpha_{2}\right)-\left[\bar{\alpha}_{1}^{2}\left(\beta_{1}-\beta_{2}\right)+c_{1}^{2}\left(\alpha_{1}-\alpha_{2}\right)\right] v_{0}=0,
$$

from which we see that $v_{0}$ is an algebroid function of degree at most 16. Moreover, it is seen that none of $0,\left(\alpha_{2}-\beta_{1}\right) /\left(\alpha_{2}-\alpha_{1}\right)$, and $\left(\beta_{2}-\beta_{1}\right) /\left(\beta_{2}-\alpha_{1}\right)$ is a root of (6.55), for otherwise we get $\bar{\alpha}_{1} \equiv 0$, a contradiction. If $v_{0} \equiv 0$, then for a point $z_{0}$ such that $f\left(z_{0}+1\right)=\alpha_{1}\left(z_{0}+1\right)$, we have $u\left(z_{0}\right)=v\left(z_{0}\right)=0$ and from (6.53) we see 
that $c_{1}\left(z_{0}\right) u\left(z_{0}\right) / v\left(z_{0}\right)=\infty$, a contradiction since $\bar{f}=c_{1} u / v$. Therefore, $v_{0} \not \equiv 0$. Rewrite (6.54) as

$$
\frac{c_{1}^{2}\left[\left(\alpha_{1}-\alpha_{2}\right) v-\left(\beta_{1}-\alpha_{2}\right)\right] v-\left[\left(\alpha_{1}-\beta_{2}\right) v-\left(\beta_{1}-\beta_{2}\right)\right] \bar{\alpha}_{1}^{2} v^{2}}{\left[\left(\alpha_{1}-\beta_{2}\right) v-\left(\beta_{1}-\beta_{2}\right)\right] v^{2}}=\bar{f}^{2}-\bar{\alpha}_{1}^{2}
$$

From (6.52) we see that $v$ is completely ramified at poles, zeros, $\left(\alpha_{2}-\beta_{1}\right) /\left(\alpha_{2}-\alpha_{1}\right)$ points, and $\left(\beta_{2}-\beta_{1}\right) /\left(\beta_{2}-\alpha_{1}\right)$-points. This implies that (6.55) can admit only one root, for otherwise by using a similar analysis as to (5.8) in Sect. 5 we obtain from (6.56) that (6.55) has at least two roots, say, $v_{1}$ and $v_{2}$, distinct from $0, \infty,\left(\alpha_{2}-\right.$ $\left.\beta_{1}\right) /\left(\alpha_{2}-\alpha_{1}\right),\left(\beta_{2}-\beta_{1}\right) /\left(\beta_{2}-\alpha_{1}\right)$ such that $v$ is completely ramified at $v_{1}$-points and $v_{2}$-points, which is impossible by Theorem 1 . Therefore, the discriminant of the quadratic Eq. (6.55) with respect to $v_{0}$ satisfies

$$
\left[\bar{\alpha}_{1}^{2}\left(\beta_{1}-\beta_{2}\right)+c_{1}^{2}\left(\alpha_{1}-\alpha_{2}\right)\right]^{2}-4 \bar{\alpha}_{1}^{2} c_{1}^{2}\left(\alpha_{1}-\beta_{2}\right)\left(\beta_{1}-\alpha_{2}\right)=0,
$$

i.e.,

$$
\bar{\alpha}_{1}^{4}-\frac{2 c\left[2\left(\beta_{1}-\alpha_{2}\right)\left(\alpha_{1}-\beta_{2}\right)-\left(\beta_{1}-\beta_{2}\right)\left(\alpha_{1}-\alpha_{2}\right)\right]}{\left(\beta_{1}-\beta_{2}\right)^{2}} \bar{\alpha}_{1}^{2}+\frac{c^{2}\left(\alpha_{1}-\alpha_{2}\right)^{2}}{\left(\beta_{1}-\beta_{2}\right)^{2}}=0 .
$$

Similarly, we obtain that (6.57) still holds when replacing $\bar{\alpha}_{1}$ by any of $\bar{\alpha}_{2}, \bar{\beta}_{1}$, and $\bar{\beta}_{2}$, which implies that $\bar{\alpha}_{1}^{2}=\bar{\alpha}_{2}^{2}$ or $\bar{\alpha}_{1}^{2}=\bar{\beta}_{1}^{2}$. Thus we need to consider the following two cases: (1), $\alpha_{1}=-\alpha_{2}, \beta_{1}=-\beta_{2}$; and (2), $\alpha_{1}=-\beta_{1}, \alpha_{2}=-\beta_{2}$.

If $\alpha_{1}=-\alpha_{2}, \beta_{1}=-\beta_{2}$, then by Vieta's formulas we obtain from (6.57) that

$$
\bar{\alpha}_{1}^{2} \bar{\beta}_{1}^{2}=\frac{c^{2} \alpha_{1}^{2}}{\beta_{1}^{2}}, \quad \bar{\alpha}_{1}^{2}+\bar{\beta}_{1}^{2}=c \frac{\beta_{1}^{2}+\alpha_{1}^{2}}{\beta_{1}^{2}}
$$

Set $\gamma_{1}=\alpha_{1} / \beta_{1}, \gamma_{1}^{2} \not \equiv 0,1$ and $\gamma_{2}=\theta \bar{\gamma}_{1} / \gamma_{1}=c / \bar{\beta}_{1}^{2}, \theta= \pm 1$. Then $\gamma_{1}^{2}$ is meromorphic and $\gamma_{2}$ satisfies $\left(\bar{\gamma}_{1}^{2}+1\right) \gamma_{1}=\theta \bar{\gamma}_{1}\left(\gamma_{1}^{2}+1\right)$, which gives $\bar{\gamma}_{1}=\theta \gamma_{1}$ or $\bar{\gamma}_{1} \gamma_{1}=\theta$. It follows that $\gamma_{2} \equiv 1$ or $\gamma_{2}=\bar{\gamma}_{1}^{2}$. If $\gamma_{2} \equiv 1$, then $\gamma_{1}^{2}$ is a periodic function with period 1. By doing the transformation $f \rightarrow \beta_{1} f$, (6.51) becomes

$$
\bar{f}^{2}=\frac{f^{2}-\gamma_{1}^{2}}{f^{2}-1}
$$

which is the Eq. (3.10); if $\gamma_{2}=\bar{\gamma}_{1}^{2}$, then $\bar{\gamma}_{1}^{2} \gamma_{1}^{2}=1$. By doing the transformation $f \rightarrow \beta_{1} f$, (6.51) becomes

$$
\bar{f}^{2}=\frac{\bar{\gamma}_{1}^{2} f^{2}-1}{f^{2}-1}
$$

which is the Eq. (3.11). 
If $\alpha_{1}=-\beta_{1}, \alpha_{2}=-\beta_{2}$, then by Vieta's formulas we obtain from (6.57) that

$$
\bar{\alpha}_{1}^{2} \bar{\alpha}_{2}^{2}=c^{2}, \quad \bar{\alpha}_{1}^{2}+\bar{\alpha}_{2}^{2}=2 c \frac{-2\left(\alpha_{1}+\alpha_{2}\right)^{2}+\left(\alpha_{1}-\alpha_{2}\right)^{2}}{\left(\alpha_{1}-\alpha_{2}\right)^{2}} .
$$

Set $\lambda=\left(\alpha_{1} / \alpha_{2}\right)^{1 / 2}, \lambda^{2} \not \equiv 0, \pm 1$. Let $d=\lambda+1 / \lambda$. Then $d^{2}=\left(\alpha_{1}+\alpha_{2}\right)^{2} / \alpha_{1} \alpha_{2}$ is a meromorphic function satisfying

$$
\bar{d}^{2}-2=2 \theta \frac{-2 d^{2}+d^{2}-4}{d^{2}-4}
$$

i.e.,

$$
\bar{d}^{2}\left(d^{2}-4\right)=2(1-\theta) d^{2}-8(1+\theta)
$$

where $\theta= \pm 1$. By doing the transformation $f \rightarrow\left(\alpha_{1} \alpha_{2}\right)^{1 / 2} f,(6.51)$ becomes

$$
\bar{f}^{2}=\theta \frac{(f-\lambda)\left(f-\lambda^{-1}\right)}{(f+\lambda)\left(f+\lambda^{-1}\right)}=\theta \frac{f^{2}-d f+1}{f^{2}+d f+1},
$$

which is the Eq. (3.12).

\section{3 $Q(z, f)$ has Three Distinct Roots}

In this case, $f$ is completely ramified at $\beta_{j}$-points. By using a similar analysis on the $\beta_{j}$-points of $f$ as on the $\alpha_{i}$-points of $f$ in (5.2) in Sect. 5, we obtain the following three possibilities:

$$
\begin{aligned}
\bar{f}^{3} & =\frac{c\left(f-\alpha_{1}\right)^{3}}{\left(f-\beta_{1}\right)\left(f-\beta_{2}\right)\left(f-\beta_{3}\right)}, \\
\bar{f}^{4} & =\frac{c\left(f-\alpha_{1}\right)^{4}}{\left(f-\beta_{1}\right)^{2}\left(f-\beta_{2}\right)\left(f-\beta_{3}\right)}, \\
\bar{f}^{6} & =\frac{c\left(f-\alpha_{1}\right)^{6}}{\left(f-\beta_{1}\right)^{3}\left(f-\beta_{2}\right)^{2}\left(f-\beta_{3}\right)},
\end{aligned}
$$

where $\alpha_{1}$ is meromorphic. In (6.58), $f$ is threefold ramified at $\beta_{j}$-points, $j=1,2,3$. This implies that $\beta_{j} \not \equiv 0, j=1,2,3$, for otherwise $f$ will also be threefold ramified at $\alpha_{1}$-points, which yields a contradiction to (2.4). Similarly, in Eqs. (6.59)-(6.60), we have $\beta_{j} \not \equiv 0, j=1,2,3$. By doing a bilinear transformation $f \rightarrow 1 / f$, if $\alpha_{1} \equiv 0$ we obtain Eqs. (5.16) $-(5.18)$ and if $\alpha_{1} \not \equiv 0$ we obtain Eqs. (6.17)-(6.19). This completes the proof of Theorem 2 .

Acknowledgements Open access funding provided by University of Eastern Finland (UEF) including Kuopio University Hospital. 
Open Access This article is licensed under a Creative Commons Attribution 4.0 International License, which permits use, sharing, adaptation, distribution and reproduction in any medium or format, as long as you give appropriate credit to the original author(s) and the source, provide a link to the Creative Commons licence, and indicate if changes were made. The images or other third party material in this article are included in the article's Creative Commons licence, unless indicated otherwise in a credit line to the material. If material is not included in the article's Creative Commons licence and your intended use is not permitted by statutory regulation or exceeds the permitted use, you will need to obtain permission directly from the copyright holder. To view a copy of this licence, visit http://creativecommons.org/licenses/by/4.0/.

\section{References}

1. Ablowitz, M.J., Halburd, R., Herbst, B.: On the extension of the Painlevé property to difference equations. Nonlinearity 13(3), 889-905 (2000)

2. Baker, I.N.: On a class of meromorphic functions. Proc. Am. Math. Soc. 17, 819-822 (1966)

3. Bank, S.B., Kaufman, R.P.: On the growth of meromorphic solutions of the differential equation $\left(y^{\prime}\right)^{m}=R(z, y)$. Acta Math. 144(3-4), 223-248 (1980)

4. Baxter, R.J.: Exactly Solved Models in Statistical Mechanics. Academic Press, Inc., London (1982)

5. Gross, F.: Erratum: “On the equation $f^{n}+g^{n}=1$ ”. Bull. Am. Math. Soc. 72, 576 (1966)

6. Gross, F.: On the equation $f^{n}+g^{n}=1$. Bull. Am. Math. Soc. 72, 86-88 (1966)

7. Halburd, R.G., Korhonen, R.J.: Finite-order meromorphic solutions and the discrete Painlevé equations. Proc. Lond. Math. Soc. (3) 94(2), 443-474 (2007)

8. Halburd, R.G., Korhonen, R.J.: Meromorphic solutions of difference equations, integrability and the discrete Painlevé equations. J. Phys. A 40(6), R1-R38 (2007)

9. Halburd, R.G., Korhonen, R.J., Tohge, K.: Holomorphic curves with shift-invariant hyperplane preimages. Trans. Am. Math. Soc. 366(8), 4267-4298 (2014)

10. Hayman, W.K.: Meromorphic Functions. Oxford Mathematical Monographs. Clarendon Press, Oxford (1964)

11. Katajamäki, K.: Algebroid solutions of binomial and linear differential equations. Ann. Acad. Sci. Fenn. Ser. A I Math. Dissertationes, No. 90, 48 pp (1993)

12. Laine, I.: On the behaviour of the solutions of some first order differential equations. Ann. Acad. Sci. Fenn. Ser. A I 497, 26 pp (1971)

13. Laine, I.: Nevanlinna Theory and Complex Differential Equations. De Gruyter Studies in Mathematics, vol. 15. Walter de Gruyter \& Co., Berlin (1993)

14. Malmquist, J.: Sur les fonctions a un nombre fini de branches définies par les équations différentielles du premier ordre. Acta Math. 36(1), 297-343 (1913)

15. Mohon'ko, A.Z.: The Nevanlinna characteristics of certain meromorphic functions. Teor. Funktsii Funktsional. Anal. i Prilozhen 14, 83-87 (1971)

16. Nakamura Y., Yanagihara N.: Meromorphic solutions of some polynomial difference equations. Analytic function theory of one complex variable, Pitman Res. Notes Math. Ser., vol. 212, Longman Sci. Tech., Harlow, pp. 178-198 (1989)

17. Quispel, G.R.W., Roberts, J.A.G., Thompson, C.J.: Integrable mappings and soliton equations. Phys. Lett. A 126(7), 419-421 (1988)

18. Quispel, G.R.W., Roberts, J.A.G., Thompson, C.J.: Integrable mappings and soliton equations. II. Phys. D 34(1-2), 183-192 (1989)

19. Ramani, A., Carstea, A.S., Grammaticos, B., Ohta, Y.: On the autonomous limit of discrete Painlevé equations. Phys. A 305(3-4), 437-444 (2002)

20. Selberg, H.L.: Über eine Eigenschaft der logaritmischen Ableitung einer meromorphen oder algebroiden Funktion endlicher Ordnung. Avhandlinger Oslo, vol. 14 (1929)

21. Selberg, H.L.: Über die Wertverteilung der algebroiden Funktionen. Math. Z. 31, 709-728 (1930)

22. Selberg, H.L.: Algebroide Funktionen und Umkehrfunktionen Abelscher Integrale. Avh. Norske Vid. Akad. Oslo 8, 1-72 (1934)

23. Shimomura, S.: Entire solutions of a polynomial difference equation. J. Fac. Sci. Univ. Tokyo Sect. IA Math. 28(2), 253-266 (1981)

24. Steinmetz, N.: Eigenschaften eindeutiger Lösungen gewöhnlicher Differentialgleichungen im Komplexen. Dissertation, Karlsruhe Univ., Karlsruhe (1978) 
25. Ullrich, E.: Über den Einfluß der Verzweigtheit einer Algebroide auf ihre Wertverteilung. J. Reine Angew. Math. 167, 198-220 (1931)

26. Valiron, G.: Sur la dérivée des fonctions algébroïdes. Bull. Soc. Math. France 59, 17-39 (1931)

27. Yamanoi, K.: The second main theorem for small functions and related problems. Acta Math. 192(2), 225-294 (2004)

28. Yanagihara, N.: Meromorphic solutions of some difference equations. Funkcial. Ekvac. 23(3), 309-326 (1980)

29. Yanagihara, N.: Meromorphic solutions of some difference equations of $n$th order. Arch. Ration. Mech. Anal. 91(2), 169-192 (1985)

30. Yanagihara N.: Polynomial difference equations which have meromorphic solutions of finite order. Analytic function theory of one complex variable. Pitman Res. Notes Math. Ser., vol. 212, Longman Sci. Tech., Harlow, pp. 368-392 (1989)

31. Yosida, K.: A generalization of Malmquist's theorem. J. Math. 9, 253-256 (1933)

Publisher's Note Springer Nature remains neutral with regard to jurisdictional claims in published maps and institutional affiliations.

\section{Affiliations}

\section{Risto Korhonen ${ }^{1} \cdot$ Yueyang Zhang ${ }^{2,3}$}

$凶$ Risto Korhonen

risto.korhonen@uef.fi

Yueyang Zhang

zhangyueyang@ustb.edu.cn

1 Department of Physics and Mathematics, University of Eastern Finland, P.O. Box 111, 80101 Joensuu, Finland

2 School of Mathematics and Physics, University of Science and Technology Beijing, No. 30 Xueyuan Road, Haidian, Beijing 100083, People's Republic of China

3 LMIB and School of Mathematics and Systems Science, Beihang University, No. 37 Xueyuan Road, Haidian, Beijing 100191, People's Republic of China 